\title{
Double Overexpression of Mir-19a \& Mir-20a in Induced Pluripotent Stem Cell-Derived Mesenchymal Stem Cells Effectively Preserves the Left Ventricular Function tn Dilated Cardiomyopathic Rat
}

Jiunn-Jye Sheu

Chang Gung Memorial Hospital Kaohsiung Branch

Han-Tan Chai

Chang Gung Memorial Hospital Kaohsiung Branch

Pei-Hsun Sung

Chang Gung Memorial Hospital Kaohsiung Branch

John Y. Chiang

National Sun Yat-sen University

Tien-Hung Huang

Chang Gung Memorial Hospital Kaohsiung Branch

Pei-Lin Shao

Asia University

Shun-Cheng Wu

Kaohsiung Medical University

Hon-Kan Yip ( $\nabla$ han.gung@msa.hinet.net)

Chang Gung Memorial Hospital Kaohsiung Branch https://orcid.org/0000-0002-6305-5717

\section{Research}

Keywords: dilated cardiomyopathy, double overexpression of microRNAs, oxidative stress, inflammation, mitochondrial damage

Posted Date: May 12th, 2021

DOI: https://doi.org/10.21203/rs.3.rs-504467/v1

License: (c) (1) This work is licensed under a Creative Commons Attribution 4.0 International License.

Read Full License 


\section{Abstract}

Background: This study tested the hypothesis that double overexpression of miR-19a \& miR-20a (dOexmIRs) in human induced pluripotent stem cell (iPS)-derived mesenchymal stem cells (MSCs) effectively preserved left-ventricular-ejection-fraction (LVEF) in dilated cardiomyopathy (DCM) (i.e., induced by doxorubicin) rat.

Methods and Results: In vitro study was categorized into groups G1 (iPS-MSC), G2 (iPS-MSC ${ }^{\text {dOex-mIRs }}$ ), G3 (iPS-MSC $+\mathrm{H}_{2} \mathrm{O}_{2} / 100 \mathrm{uM}$ ), and $\mathrm{G} 4$ (iPS-MSC ${ }^{\text {dOex-mIRs }}+\mathrm{H}_{2} \mathrm{O}_{2} / 100 \mathrm{uM}$ ). The in vitro results showed the cell viability was significantly lower in $\mathrm{G} 3$ than in $\mathrm{G} 1$ and $\mathrm{G} 2$, and that was reversed in $\mathrm{G} 4$ but it showed no difference between $\mathrm{G} 1 / \mathrm{G} 2$ at time points of $6 \mathrm{~h} / 24 \mathrm{~h} / 48 \mathrm{~h}$, whereas the flow cytometry of intracellular/mitochondrial oxidative stress (DCFA/mitoSOX) and protein expressions of mitochondrialdamaged (cytosolic-cytochrome-C/DRP1/Cyclophilin-D), oxidative-stress (NOX-1/NOX2), apoptotic (cleaved-caspase-3/PARP), fibrotic ( $p$-Smad3/TGF- $ß$ ) and autophagic (ratio of LC3B-II/LC3BI) biomarkers exhibited an opposite pattern of cell-proliferation rate (all $p<0.001)$. Adult-male SD rats $(n=32)$ were equally divided into groups 1 (sham-operated control), 2 (DCM), 3 (DCM + iPS-MSCs/1.2 $\times 10^{6}$

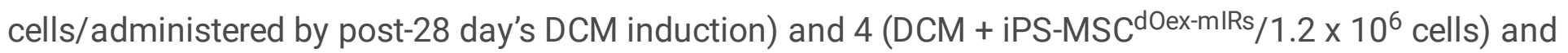
euthanized by day 60 after DCM induction. LV myocardium protein expressions of oxidative-stress signaling (p22-phox/NOX-1/NOX-2/ASK1/p-MMK4,7/p-JNK1,2/p-cJUN), upstream (TLR4/MAL/MyD88/TRIF/TRAM/ TFRA6/IKK ${ }_{\alpha / \beta} /$ NF-KB) and downstream (TNF-a/IL-1ß/MMP-9) inflammatory signalings, apoptotic (cleaved-PARP/mitochondrial-Bax), fibrotic (Smad3/TGF-ß), mitochondrial-damaged (cytosolic-cytochrome-C/DRP1/cyclophilin-D) and autophagic (beclin1/Atg5) biomarkers were highest in group 2, lowest in group 1 and significantly lower in group 4 than in group 3 , whereas the LVEF exhibited an opposite pattern of oxidative stress (all $p<0.0001)$.

Conclusion: iPS-MSC ${ }^{\text {dOex-mIRs }}$ therapy was superior to iPS-MSC therapy for preserving LV function in DCM rat.

\section{Introduction}

Assuredly, idiopathic dilated cardiomyopathy (DCM), a primary non-ischemic cardiac muscle disease, is clearly recognized as the consequence of systolic dysfunction and dilatation of chamber size of the left or both of ventricles [1, 2]. In fact, vast data have revealed that this disease accounts for approximately one-third of heart failure (HF) patients and is associated with adverse clinical outcomes and unacceptable high morbidity and mortality [3-8]. Abundant clinical trials and clinical observation studies have shown that HF, caused by different disease entity, is an important cause of morbidity and mortality in industrialized countries, with an estimation up to 5.7 million people affected in the United States $[9,10]$ and 30-50 million patients worldwide [11], and 5-year mortality reaching 50\% [12].

Despite remarkable progress has been established in both advanced pharmacological and nonpharmacological modalities to treat DCM-related HF, the number of patients for hospitalization and 
deaths of HF patient has increased steadily [13-17]. The aforementioned issues [12-16] highlight that the treatment of HF caused by DCM is currently an unmet need, suggesting that to develop a safe and efficacious strategic management for DCM is utmost important and urgent to patients and physicians.

Inflammation is believed as a distinctive hallmark of HF $[18,19]$ in circulation and proinflammatory cytokines in myocardium [18, 20-22]. This inflammatory response has been further identified in damaged myocardium during propagation of chronic HF to promote monocyte activation and the further production of cytokines, thus augmenting cardiac dysfunction [23], resulting in myocardial fibrosis and cardiac remodeling [24]. Additionally, studies have previously further displayed that an increased reactive oxygen species (ROS) plays a crucial role in the sudden death of these HF/DCM patients [25-27].

Furthermore, plentiful clinical and experimental studies have shown that mitochondrial dysfunction plays a principal role in the histopathological process of heart disease [28-30].

Recently, the utilization of human induced pluripotent stem cell derived mesenchymal stem cells (iPSCMSCs) has emerged as an innovative option for regenerative medicine [31, 32] and as a therapeutic alternative for various disease entities [33-35] mainly through suppressing the inflammation and generation of oxidative stress as well as the immunogenicity [36]. However, a full investigation of the impact of iPSC-MSCs on DCM has not been undertaken. Additionally, we have recently identified that the circulating levels of five anti-apoptotic micro-RNAs (i.e., miR-374a-5p, miR-19a-3p, miR-106b-5p, miR-26b$5 p$, and miR-20a-5p) were significantly lower in chronic kidney disease (CKD) patients than in healthy subjects [37]. These aforementioned issues raised the hypothesis that double overexpression of miR-19a \& miR-20a (dOex-mIRs) in induced pluripotent stem cell (iPS)-derived mesenchymal stem cells (MSCs) (i.e., iPS-MSC ${ }^{\text {dOex-mIRs }}$ ) might effectively preserve heart function in DCM rat.

\section{Materials And Methods}

\section{Ethics}

All animal procedures were approved by the Institute of Animal Care and Use Committee at Kaohsiung Chang Gung Memorial Hospital (Affidavit of Approval of Animal Use Protocol No. 2019061902) and performed in accordance with the Guide for the Care and Use of Laboratory Animals.

Animals were housed in an Association for Assessment and Accreditation of Laboratory Animal Care International (AAALAC; Frederick, MD, USA)-approved animal facility in our hospital with controlled temperature and light cycles $\left(24^{\circ} \mathrm{C}\right.$ and $12 / 12$ light cycle).

\section{Methodology of in vitro study of cell culturing for differentiation of human iPSC into mesenchymal stem cells (MSCs)}

The procedure and protocol of human iPSC culture for differentiation into MSCs have been described in our previous study [36] and detailed in formation was illustrated as supplementary Figure 1. In details, at day 1 , the human iPSCs [mTeSR ${ }^{\text {TM }} 1$; StemCell, \#28315) were first washed by $5 \mathrm{~mL}$ PBS, followed by $2 \mathrm{~mL}$ 
Accutase (Gibco, \#A1110501; Accutase: PBS = 1:1); the incubator reaction continued for $1 \mathrm{~min}$. The $2 \mathrm{~mL}$ KO DMEM/F12 (Gibco, \#12660012) was added and the cells were collected in $15 \mathrm{~mL}$ centrifuge tubes for 5 -minute duration of centrifuge (x200 g). The cells were then cultured in a 10-cm dish for $24 \mathrm{~h}$ in $\mathrm{mTeSR}^{\mathrm{TM}} 1$ culture medium.

By day 2 , the cells ( $\mathrm{mTeSR}^{\mathrm{TM}} 1$ ) were collected and washed by $5 \mathrm{~mL}$ PBS. STEMdiff ${ }^{{ }^{\mathrm{T} M}}{ }_{\text {-ACF Mesenchymal }}$ Induction Medium (StemCell, \#05241) was added to incubator culture and proceeded for $24 \mathrm{~h}$. The STEMdiff ${ }^{\mathrm{TM}}$-ACF Mesenchymal Induction Medium was exchanged once per day from days 1 to 3 . This procedure was repeated on days 3 to 6 . On days 7 to 21 , the procedure was repeated but the culture medium was refreshed every 3 days.

\section{miR-19a-3p and miR-20a-5p were candidates for double overexpression in iPS-MSCs (iPS-MSC ${ }^{\text {dOex-mIRs }}$ ) and treatment of DCM in rodent}

The procedure and protocol were based on our recent report [38]. We had identified that miR-19a-3p and miR-20a-5p were the two most suitable candidates among the five miRNAs (i.e., miR-374a-5p/ miR-19a$3 p / m i R-106 b-5 p / m i R-26 b-5 p / m i R-20 a-5 p$ ) to be overexpressed (i.e., transfection) for the purpose of treatment of chronic kidney disease + ischemia-reperfusion animals [38]. In detail, transfections of miR19a-3p and miR-20a-5p mimics efficiently augmented the miRNA expressions and further decreased related gene expressions. Transfections of mimics ( $25 \mathrm{nM})$ were conducted with TransIT-X2 Dynamic Delivery System (Mirus), by following the manufacturer's instruction. The iPS-MSCs were recognized $>80 \%$ confluence on the day of transfection. TransIT-X2 reagent was mixed with miRNA mimics for 25 minutes at room temperature. The miRNA mimics-containing complexes were further distributed into cells. Two days later, relevant expressions of miRNAs and genes were validated by the real-time qPCR assay.

\section{DCM induction in rodent by doxorubicin (Dox) and animal grouping}

Pathogen-free, adult male Sprague-Dawley (SD) rats ( $\mathrm{n}=32$ ) weighing 320-350 g (Charles River Technology, BioLASCO Taiwan Co. Ltd., Taiwan) were utilized in this study. The procedure and protocol of Dox-induced rodent DCM model have been described in detail in our previous report [30]. In detail, the accumulated dose of $12.5 \mathrm{mg}$ per $\mathrm{kg}$ at 4 separated time points within 20 days (i.e., once every 5 days) in each rat by intraperitoneal (IP) administration was applied in the present study.

Animals were equally categorized into group 1 (sham-control, i.e., by IP administration of $1.0 \mathrm{cc}$ saline four times within 20 days, followed by opening chest wall only at day 28 after DCM induction), group 2 (DCM only), group 3 [DCM + iPS-MSCs/ $1.2 \times 10^{6}$ cells/administered by day 28 after DCM induction) and

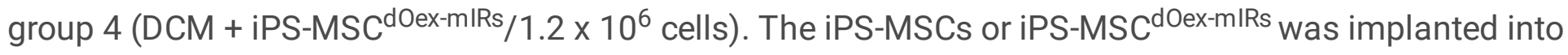
the LV myocardium via opening the chest wall after intubation with animal ventilatory support by day 28 after DCM induction by Dox. Additionally, the dosage of MSCs in the present study was based on our 
previous studies $[36,39]$. In the current study, the animals in each group were euthanized by day 60 and the heart specimen was harvested in each animal for individual study.

\section{Determinant LVEF by utilizing the transthoracic echocardiography}

Transthoracic echocardiography was performed in each group prior to and on days 28 and 60 after DCM induction. The procedure was performed by an animal cardiologist blinded to this experimental design using an ultrasound machine (Vevo 2100, Visualsonics). M-mode standard two-dimensional (2D) left parasternal-long axis echocardiographic examinations were conducted. Left ventricular (LV) internal dimensions [end-systolic diameter (ESD) and end-diastolic diameter (EDD)] were measured at the mitral valve level of the left ventricle, according to the leading-edge method of American Society of Echocardiography, by using at least three consecutive cardiac cycles. The left ventricular ejection fraction $\left(\right.$ LVEF) was calculated as follows: LVEF $(\%)=\left[\left(\right.\right.$ LVEDD $^{3}-$ LVEDS $\left.^{3}\right) /$ LVEDD $\left.^{3}\right] \times 100 \%$.

\section{Western blot analysis of LV myocardium}

The procedure and protocol have been described in detail in our previous reports [36-39].Briefly, primary antibodies against tumor necrosis factor (TNF)- $a$ (1: 1000, Cell Signaling), nuclear factor (NF)-KB (1:1000, Abcam), tumor necrosis factor receptor-associated factor 6 (TRAF6) (1:2000, Abcam), toll-like receptor (TLR)-4 (1:1000, Novus), myeloid differentiation primary response 88 (MyD88) (1:1000, Abcam), myelin and lymphocyte protein (Mal) (1:1000, Abcam), TRIF (1:1000, Abcam), translocating chain-associated membrane protein (TRAM) (1:1000, Thermo Fisher Scientific), TNF receptor associated factor 6 (TRAF6) (1:2000, Abcam), IKK-a (1:5000, Abcam), IKK-ß (1:1000, Cell Signaling), nuclear factor of kappa light polypeptide gene enhancer in B-cells inhibitor, alpha (IKB-a) (1:1000, Cell Signaling), apoptosis signalregulating kinase 1 (ASK1) (1:1000, Abcam), phosphorylated mitogen-activated protein kinase 4 ( $\mathrm{p}$ MMK4) (1:1000, Cell Signaling), p-MMK7 (1:1000, Thermo Fisher Scientific), p-JNK1/2 (1:1000, Abcam), p-cJUN (1:1000, Abcam), Atg5 (1:1000, Cell Signaling), Beclin1 (1:1000, Cell Signaling), interleukin (IL)-1ß (1:1000, Cell Signaling), matrix metalloproteinase (MMP)-9 (1:2000, Abcam), NOX-1 (1:1500, SigmaAldrich), NOX-2 (1:1000, Sigma-Aldrich), cytosolic cytochrome C (1:2000, BD), cyclophilin-D (1:3000, Abcam), dynamin-related protein 1 (DRP1) (1:1000, Cell Signaling), LC3B-II (1:2000, Abcam), LC3B-I (1:2000, Abcam), mitochondrial Bax (1:1000, Abcam), cleaved caspase 3 (1:1000, Cell Signaling), cleaved Poly (ADP-ribose) polymerase (c-PARP) (1:1000, Cell Signaling), Smad3 (1:1000, Cell Signaling) and transforming growth factor (TGF)- $ß$ (1:1000, Abcam) were used. Signals were detected with horseradish peroxidase (HRP)-conjugated goat anti-mouse, goat anti-rat, or goat anti-rabbit lgG.

Immunoreactive bands were visualized by enhanced chemiluminescence (ECL; Amersham Biosciences), which was then exposed to Biomax L film (Kodak). For quantification, ECL signals were digitized using Labwork software (UVP).

\section{Immunohistochemical (IHC) and immunofluorescent (IF) studies}


The procedures and protocols for IHC and IF examinations were based on our previous reports [36-39]. Briefly, for IHC and IF staining, rehydrated paraffin sections were first treated with $3 \% \mathrm{H}_{2} \mathrm{O}_{2}$ for 30 minutes and incubated with Immuno-Block reagent (BioSB, Santa Barbara, CA, USA) for 30 minutes at room temperature. Sections were then incubated with primary antibodies specifically against $Y-H 2 A X(1: 1000$, Abcam) and CD14 (1:200, Thermo Fisher), while sections incubated with the use of irrelevant antibodies served as controls. Three sections of heart specimens from each rat were analyzed. For quantification, three randomly selected HPFs (400x for IF studies) were analyzed in each section.

\section{Histopathological finding of myocardial fibrosis}

The procedure and protocol were based on our previous studies [30]. In details, hematoxylin and eosin and Masson's trichrome staining were utilized for identification of LV fibrotic area. Three serial sections of LV myocardium in each animal were prepared at $4 \mu \mathrm{m}$ thickness by Cryostat (Leica CM3050S). The integrated area $\left(\mu \mathrm{m}^{2}\right)$ of fibrosis on each section were calculated using the Image Tool 3 (IT3) image analysis software (University of Texas, Health Science Center, San Antonio, UTHSCSA; Image Tool for Windows, Version 3.0, USA). Three randomly selected high-power fields (HPFs) (100 x) were analyzed in each section. After determining the number of pixels in each fibrotic area per HPF, the numbers of pixels obtained from three HPFs were calculated. The procedure was repeated in two other sections of each animal. The mean pixel number per HPF for each animal was then analyzed by calculating all pixel numbers and dividing by 9 . The mean integrated area $\left(\mu \mathrm{m}^{2}\right)$ of fibrosis in LV myocardium per HPF was obtained using a conversion factor of 19.24 (since $1 \mu \mathrm{m}^{2}$ represents 19.24 pixels).

\section{MTT assay, qPCR analysisand flow cytometric analysis for identification of total cellular and mitochondrial oxidative stress and membrane potential of mitochondria in iPS-MSCs}

For the purposes of in vitro study, the culturing cells were categorized into G1 (iPS-MSC), G2 (iPSMSC ${ }^{\text {dOex-mIRs }}$ ), G3 (iPS-MSC $+\mathrm{H}_{2} \mathrm{O}_{2} / 100 u M$ ), and G4 (iPS-MSC ${ }^{\text {dOex-mIRs }}+\mathrm{H}_{2} \mathrm{O}_{2} / 100 \mathrm{uM}$ ), respectively. The cells were finally collected for the flow cytometric analysis for assessment of total cellular (i.e., by $\mathrm{H}_{2}$ DCFDA test) and mitochondrial (i.e., by Mito-SOX assay) oxidative stress and membrane potential of mitochondria [i.e., Tetramethylrhodamine, Ethyl Ester, Perchlorate (TMRE assay)].

Additionally, the MTT assay was utilized in the present study to determine the cellular metabolic activity as an indicator of cell viability, proliferation, and cytotoxicity.

Furthermore, the cells were also collected after culturing for Western blot analysis. Finally, qPCR analysis was utilized to assess the success of overexpression of iPS-MSC ${ }^{\text {ex-mIRs. }}$.

\section{Procedure and protocol for measurement of reactive oxygen species (ROS)}

The procedure and protocol have been described in our previous report [40]. In detail, the 2',7'dichlorodihydrofluorescin diacetate $\left(\mathrm{H}_{2}\right.$ DCFDA, Molecular Probes) was dissolved in DMSO at a concentration of $25 \mathrm{mg} / \mathrm{ml}$. After dilution with $50 \%$ ethanol to a final concentration of $2.5 \mathrm{mg} / \mathrm{ml}, 6 \mu \mathrm{g}$ per 
gram for rat body weight was administered to each rat from the penis vein immediately following echocardiographic study. All animals were euthanized 30 minutes later following $\mathrm{H}_{2}$ DCFDA administration.

The fluorescence and grayscale photos were captured by utilizing the DP controller 2.1.1.183 (Olympus). Grayscale photos for measuring the fluorescence intensity were processed by using Image $\mathrm{J} 1.37 \mathrm{v}$ (National Institutes of Health, USA). Nine grayscale photos from each slide were randomly acquired. As compared with the area of increased fluorescence intensity (IFI), the baseline fluorescence intensity (BFI) (arbitrary unit/400 $\mathrm{x}$ high-power field) was defined as the area in myocardium loaded without $\mathrm{H}_{2}$ DCFDA. Six $\mathrm{BFI}$ areas were measured from each grayscale photo, from which $3 \mathrm{BFI}$ areas were randomly chosen. The mean IFI and mean BFI were then calculated. The ratio of IFI to the BFI was determined as the relative fluorescence intensity.

The LV specimen were obtained, frozen rapidly in liquid nitrogen and then stored at $-80^{\circ} \mathrm{C}$.

\section{Statistical Analysis}

Quantitative data are expressed as mean \pm SD. Statistical analyses were performed using SAS statistical software for Windows Version 8.2 (SAS Institute, Cary, NC, USA). One-way ANOVA was conducted followed by Bonferroni multiple comparison post hoc test for comparing variables among groups. $A$ probability value $<0.05$ was considered statistically significant.

\section{Results}

\section{The results of in vitro studies (Figures 1, 2, 3 and 4)}

To elucidate the cellular viability, the MTT assay was utilized. The result showed that this parameter was significantly higher in G1 (iPS-MSC) and G2 (iPS-MSC ${ }^{\text {dOex-mIRs) }}$ ) than in G3 (iPS-MSC $+\mathrm{H}_{2} \mathrm{O}_{2} / 100 \mathrm{uM}$ ) and G4 (iPS-MSC dOex-mIRs $+\mathrm{H}_{2} \mathrm{O}_{2} / 100 \mathrm{uM}$ ), and significantly higher in $\mathrm{G} 4$ than in $\mathrm{G} 3$ (Fig. 1), suggesting that overexpression of double microRNAs (i.e., miR-19a-3p and miR-20a-5p) was more resistant to oxidative stress damage.

Next, we assessed the capacity of microRNA transfection into iPS-MSCs by performing the relative quantitative qPCR. The result showed that relative miR-19a-3p and miR-20a-5p were lowest in G3, highest in $\mathrm{G} 2$ and significantly higher in $\mathrm{G} 4$ than in $\mathrm{G} 1$ (Fig. 1), suggesting that not only the miRs were successfully transfected into iPS-MSCs but also further proved that the dOex-mIRs still had good capacity of transfection into iPS-MSC even in the situation of $\mathrm{H}_{2} \mathrm{O}_{2}$ treatment (i.e., oxidative stress).

Furthermore, to clarify whether the iPS-MSC ${ }^{\text {dOex-mIRs }}$ could offer better ability to protect the cells against the increments of oxidative stress in cellular and mitochondrial levels as well as the active mitochondria

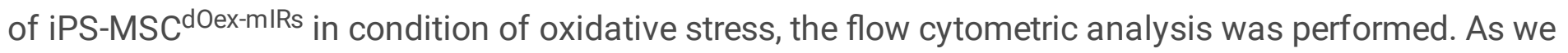
expected, the fluorescent intensity of DCFDA (i.e., an indicator of total intracellular oxidative stress) and 
Mito-SOX (i.e., an indicator of mitochondrial oxidative stress were highest in G3 than in G1 and G2, and those were significantly reversed in $\mathrm{G} 4$, whereas these parameters did not differ between $\mathrm{G} 1$ and $\mathrm{G} 2$ (Fig. 2). On the other hand, the TMRE, an index of membrane potential of mitochondria, was highest in $G 2$, lowest in G3 and significantly lower in G4 than in G1 (Fig. 2).

To delineate whether the dOex-mIRs would protect the iPS-MSCs against the $\mathrm{H}_{2} \mathrm{O}_{2}$ induced DNA damage and cellular senescence, IF microscope was utilized. The results demonstrated the cellular expression of $Y$-H2AX (Fig. 2), an indicator of DNA damage, and the positively-stained $\beta$-galactosidase (Fig. 4) cells were significantly higher in $\mathrm{G} 3$ and $\mathrm{G} 4$ than in $\mathrm{G} 1$ and $\mathrm{G} 2$ and significantly higher in $\mathrm{G} 3$ than in $\mathrm{G} 4$, but they showed no difference between $\mathrm{G} 1$ and $\mathrm{G} 2$.

Moreover, by using the Western blot analysis, we investigated the impact of dOex-mIRs on ameliorating the oxidative-stress, mitochondria-damaged, apoptotic and autophagic biomarkers in iPS-MSCs. Again as our expected, the protein expressions of NOX-1 and NOX-2 (two indicators of oxidative stress), protein expressions of cytosolic cytochrome C, cyclophilin D and DRP1 (three indices of mitochondrial-damaged parameters), protein expression of ratio of LC3BI/LC3BII (an indicator of autophagy) (Fig. 3), protein expressions of cleaved caspase 3 and cleaved PARP (two indicators of apoptosis) and protein expressions of Smad3 and TGF-1ß (two indicators of fibrosis) (Fig. 4), were significantly increased in G3 than in $\mathrm{G} 1$ and $\mathrm{G} 2$, and those were significantly reversed in $\mathrm{G} 4$, but they did not differ between $\mathrm{G} 1$ and $\mathrm{G} 2$, whereas the protein expression of mitochondrial cytochrome (Fig. 3), an indicator of mitochondrial integrity, displayed an opposite pattern of oxidative stress among the four groups.

\section{The serial changes of LVEF and fluorescent intensity of oxidative stress in LV myocardium by day 60 after DCM induction (Figure 5)}

By day 0 (i.e., at baseline), the LVEF was similar among the group 1 (sham-operated control), group 2 (DCM only), group 3 [DCM + iPS-MSCs/1.2 $\times 10^{6}$ ) and 4 (DCM + iPS-MSC dOex-mIRs $/ 1.2 \times 10^{6}$ cells). However, by day 28 after DCM induction, the LVEF was significantly higher in group 1 than in groups 2 to 4 , but it demonstrated no difference among groups 2 to 4 . On the other hand, by day 60 after DCM induction, the LVEF was highest in group 1, lowest in group 2 and significantly higher in group 4 than in group 3, implicating that iPS-MSCs effectively and iPS-MSC ${ }^{\text {dOex-mIRs }}$ further effectively preserved heart function in setting of DCM.

Additionally, the IF microscopic finding revealed that the fluorescent intensity of oxidative stress was highest in group 2, lowest in group 1 and significantly higher in group 3 than in group 4 .

\section{Cellular expressions of DNA-damaged and inflammatory biomarkers in LV myocardium by day 60 after DCM induction (Figure 6)}

The IF microscopic finding demonstrated that the protein expressions of $\mathrm{y}-\mathrm{H} 2 \mathrm{AX}+$ cells, a DNA-damaged indicator, and CD14+ cells, an indicator of inflammation, were highest in group 2, lowest in group 1 and significantly higher in group 3 than in group 4. 
Protein expressions of oxidative stress and mitochondrial damaged biomarkers in LV myocardium by day 60 after DCM induction (Figures 7 and 8)

The protein expressions of NOX-1, NOX-2 and p22 phox, three indicators of oxidative stress, were significantly lower in group 1 than in groups 2 to 4 , significantly lower in group 4 than in groups 2 and 3 and significantly lower in group 3 than in group 2 (Fig. 7). Additionally, the protein expressions of cytosolic cytochrome C, cyclophilin D and DRP1, three indicators of mitochondrial damaged biomarkers, were lowest in group 1, highest in group 2 and significantly lower in group 4 than in group 3, whereas the protein expression of mitochondrial cytochrome $\mathrm{C}$, an index of mitochondrial integrity, displayed an opposite pattern of oxidative stress among the four groups (Fig. 7). Our findings, in addition to delineating how the oxidative-stress signaling on damaging the myocardium (Fig. 8), suggested that iPSMSCs effectively and iPS-MSC ${ }^{\text {dOex-mIRs }}$ more effectively protected the mitochondria through attenuating the upregulation of oxidative stress in DCM setting.

\section{Protein expressions of mitogen-activated protein kinase (MAPK) pathway, apoptosis and autophagic biomarkers in LV myocardium by day 60 after DCM induction (Figure 9)}

We also assessed the role of MAPK in DCM setting by Western blot. As we expected, the protein expressions of apoptosis signal-regulating kinase 1 (ASK1), p-MMK4, p-MMK7, p-JNK1/2 and p-cJUN, four members of MAP kinase family, were highest in group 2, lowest in group 1 and significantly higher in group 3 than in group 4. Additionally, the protein expressions of mitochondrial Bax, cleaved caspase 3 and cleaved PARP, three indicators of apoptosis, and the protein expressions of Atg5 and Beclin1, two indices of autophagic biomarkers, displayed an identical pattern of MAPK family among the four groups.

\section{Protein expressions of upstream and downstream inflammatory signaling pathways in LV myocardium by day 60 after DCM induction (Figures 10 and 11)}

To elucidate the inflammatory signalings in LV myocardium in DCM setting, the Western blot was utilized. The result showed that the protein expressions of TLR4, MyD88, MAL, TRIF, TRAM, TRAF6, IKKa, IKKß and $\mathrm{p}-\mathrm{NF}-\mathrm{K} ß$, nine indicators of upstream signaling, and protein expressions of TNF-a, IL-1ß and MMP-9, three indices of downstream signaling, were lowest in group 1, highest in group 2 and significantly lower in group 4 than in group 3 (Fig. 10). Our findings clearly highlighted the upstream and downstream inflammatory signalings involved in initiation and propagation of myocardial damage in DCM animals (refer to Fig. 11).

\section{Discussion}

This study which investigated the therapeutic impact of dOex-mIRs of iPS-MSCs on protecting the heart against DCM damage yielded several striking implications. First, rather than only single mechanism, this study identified that the underlying mechanisms of DCM caused heart and myocardium dysfunction were quite complicated (refer to Figs. 8 and 11). Second, as compared with the SC group, the LVEF was significantly progressively worsening in DCM only, suggesting our DCM model in rodent was successfully 
created for the study. Third, the LVEF was significantly preserved by iPS-MSCs and further significantly

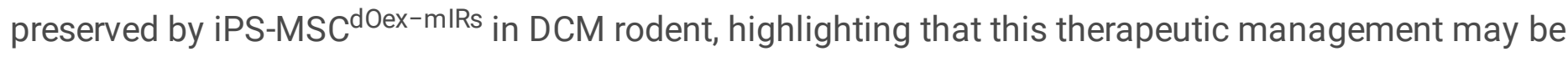
potential in the future for DCM patients, especially when their decompensated HF is refractory to conventional therapy that demands the final resort of heart transplantation.

It is well recognized that no matter how advanced pharmaceutical and accessorily mechanical devices have been utilized for those DCM patients with end-stage decompensated HF and poor LV function, the therapeutic success is still extremely limited, resulting in an unacceptably high annual mortality in these patients. Accordingly, heart transplantation, a conventional therapy, could serve as the last resort for these patients. However, the donor of living heart is extremely lacking, prompting scientists to seek an alternative modality with safety and efficacy. Intriguingly, growing data have demonstrated that cell therapy effectively improved ischemia-related organ dysfunction through tissue regeneration, angiogenesis, anti-inflammation, and oxidative stress as well as immunomodulation [31, 35-37, 39, 41, 42]. One important finding in the present study was that as compared with DCM animals, the LVEF (i.e., the heart function) was significantly preserved in iPS-MSCs treated DCM animals. Our finding corroborated with the finding of the previous studies [31, 35-37, 39, 41, 42]. The most important finding in the present study was that iPS-MSC ${ }^{\text {dOex-mIRs }}$ was expected superior to iPS-MSCs for improving the LVEF in DCM rodent. As we expected, our finding in addition to extension of the previous studies, highlights that this strategic management may pose a therapeutic potential for those DCM patients with decompensated HF and poorest heart function with requirement of heart transplantation.

It is always a universal concept that prior to offering an effective treatment for a specific disease, the delineation of the underlying mechanism of the disease entity is utmost important. An important finding in the present study was that the signaling pathway of oxidative stress, mitochondrial damaged and apoptotic biomarkers as well as the downstream members of MAPK family were identified to be markedly enhanced in DCM animals (refer to Fig. 8). Of particular importance was that not only the in vitro but also the in vivo studies demonstrated that the upregulated oxidative-stress signaling further elicited the mitochondrial damage, apoptosis and autophagic activity in iPS-MSC treated by $\mathrm{H}_{2} \mathrm{O}_{2}$ and DCM myocardium. Intriguingly, previous studies have also clearly identified that these aforementioned molecular-cellular perturbations were remarkably enhanced in DCM setting [30,43] and cardiorenal syndrome $[44,45]$ and those of MAPK family members in myocardial ischemia [46]. Accordingly, the findings of the in vitro and in vivo studies, in addition to being consistent with the findings of the previous studies [30,43-45], could, at least in part, explain why the LVEF was substantially reduced in DCM animals than in those of SC animals.

Abundant data have revealed that inflammatory activation was frequently elicited in DCM myocardium $[30,43]$, acute myocardial infarction [46] and cardiorenal syndrome [44, 45], which in turn led to progressively cardiomyocyte apoptosis and death, resulting in myocardial fibrosis and deteriorating heart function $[30,43-46]$. A principal finding in the present study was that not only the upstream but also the downstream inflammatory signalings in DCM setting were clearly delineated. In this way, our findings, in addition to strengthening the findings of previous studies [30, 43-46], further identified that the 
underlying signaling pathway of DCM was complex and probably involved in multiple signaling pathways (i.e., inflammation, oxidative stress, MAPK family and autophagy) (refer to Figs. 8 and 11). Of particularly distinctive finding was that iPS-MSC ${ }^{\text {dOex-mIRs }}$ treatment was superior to iPS-MSCs treatment for improving LVEF in DCM animals.

\section{Study limitation}

This study has limitations. First, although the study period was 60 days, the longer-term impact of iPSMSCs/ iPS-MSC ${ }^{\text {dOex-mIRs }}$ on preservation of the left ventricular function is still currently uncertain. Second, in the absence of applying $2^{\text {nd }}$ iPS-MSC ${ }^{\text {dOex-mIRs }}$, whether a $2^{\text {nd }}$ therapy would offer additional benefit on furthermore improving the cardiac function in those DCM animals remains to be answered.

In conclusion, as compared to the iPS-MSCs therapy iPS-MSC ${ }^{\text {dOex-mIRs }}$ therapy offered additional benefits on improving the LVEF in DCM animals.

\section{Declarations}

\section{Acknowledgments}

This study was supported by a program grant from Chang Gung Memorial Hospital, Chang Gung University (Grant number: CMRPG8J1111).

\section{Author Contributions}

Investigation, Jiunn-Jye Sheu, Han-Tan Chai, Pei-Hsun Sung, John Y. Chiang, Tien-Hung Huang, Pei-Lin Shao, Shun-Cheng Wu, and Hon-Kan Yip; Methodology, Jiunn-Jye Sheu, Han-Tan Chai, Pei-Hsun Sung, John Y. Chiang, Tien-Hung Huang, Pei-Lin Shao, Shun-Cheng Wu, and Hon-Kan Yip; Supervision, JiunnJye Sheu, Han-Tan Chai, Shun-Cheng Wu, and Hon-Kan Yip; Writing - review \& editing, Jiunn-Jye Sheu, Han-Tan Chai, Shun-Cheng Wu, and Hon-Kan Yip.

\section{Funding}

This study was supported by a program grant from Chang Gung Memorial Hospital, Chang Gung University (Grant number: CMRPG8J1111).

\section{Availability of data and materials}

The data that support the findings of this study are available from the corresponding authors upon reasonable request.

\section{Ethics approval and consent to participate}

All animal procedures were approved by the Institute of Animal Care and Use Committee at Kaohsiung Chang Gung Memorial Hospital (Affidavit of Approval of Animal Use Protocol No. 2019061902) and 
performed in accordance with the Guide for the Care and Use of Laboratory Animals.

\section{Consent for publication}

Not applicable.

\section{Conflicts of interest}

All authors have read the journal's policy on disclosure of potential conflicts of interest and the journal's authorship agreement. The authors declare that they have no conflicts of interest. The article has been reviewed by and approved by all named authors.

\section{References}

1. Bozkurt B, Colvin M, Cook J, et al. Current diagnostic and treatment strategies for specific dilated cardiomyopathies: a scientific statement from the American Heart Association. Circulation. 2016;134:e579-646.

2. Elliott $P$, Andersson $B$, Arbustini $E$, et al. Classification of the cardiomyopathies: a position statement from the European Society Of Cardiology Working Group on myocardial and pericardial diseases. Eur Heart J. 2008;29:270-6.

3. Maron BJ, Towbin JA, Thiene G, et al. Contemporary definitions and classification of the cardiomyopathies: an American Heart Association Scientific Statement from the Council on Clinical Cardiology, Heart Failure and Transplantation Committee; Quality of Care and Outcomes Research and Functional Genomics and Translational Biology Interdisciplinary Working Groups; and Council on Epidemiology and Prevention. Circulation. 2006;113:1807-16.

4. Felker GM, Thompson RE, Hare JM, et al. Underlying causes and long-term survival in patients with initially unexplained cardiomyopathy. N Engl J Med. 2000;342:1077-84.

5. Choi DJ, Han S, Jeon ES, et al. Characteristics, outcomes and predictors of long-term mortality for patients hospitalized for acute heart failure: a report from the korean heart failure registry. Korean Circ J. 2011;41:363-71.

6. Fuster V, Gersh BJ, Giuliani ER, et al. The natural history of idiopathic dilated cardiomyopathy. Am J Cardiol. 1981;47:525-31.

7. Juilliere $\mathrm{Y}$, Danchin N, Briancon S, et al. Dilated cardiomyopathy: long-term follow-up and predictors of survival. Int J Cardiol. 1988;21:269-77.

8. Maron BJ, Towbin JA, Thiene G, et al. Contemporary definitions and classification of the cardiomyopathies: an American Heart Association scientific statement from the council on clinical cardiology, heart failure and transplantation committee; quality of care and outcomes research and functional genomics and translational biology interdisciplinary working groups; and council on epidemiology and prevention. Circulation. 2006;113:1807-16. 
9. Lynch TL, Sivaguru IV M, et al. Oxidative Stress in Dilated Cardiomyopathy Caused by MYBPC3 Mutation. Oxid Med Cell Longev 2015; 2015:424751.

10. Holzem KM, Gomez JF, Glukhov AV, et al. Reduced response to I blockade and altered hERG1a/1b stoichiometry in human heart failure. J Mol Cell Cardiol 2015.

11. Hashimoto T, Kass DA. Stressed hearts inflame the body (in a good way). Proc Natl Acad Sci USA. 2015;112:7113-4.

12. Writing Group Members. Mozaffarian D, et al. Heart Disease and Stroke Statistics-2016 Update: A Report From the American Heart Association. Circulation 2016; 133:e38-60.

13. Yancy CW, Jessup M, Bozkurt B, et al. 2013 ACCF/AHA guideline for the management of heart failure: a report of the American College of Cardiology Foundation/American Heart Association Task Force on Practice Guidelines. J Am Coll Cardiol. 2013;62:e147-239.

14. Savarese G, Lund LH. Global Public Health Burden of Heart Failure. Card Fail Rev. 2017;3:7-11.

15. Koelling TM, Chen RS, Lubwama RN, et al. The expanding national burden of heart failure in the United States: the influence of heart failure in women. Am Heart J. 2004;147:74-8.

16. Balmforth C, Simpson J, Shen L, et al. Outcomes and Effect of Treatment According to Etiology in HFrEF: An Analysis of PARADIGM-HF. JACC Heart Fail. 2019;7:457-65.

17. Dewan P, Docherty KF, McMurray JJV. Sacubitril/Valsartan in Asian Patients with Heart Failure with Reduced Ejection Fraction. Korean Circ J. 2019;49:469-84.

18. smahil MA, Hamid T, Bansal SS, et al. Remodeling of the mononuclear phagocyte network underlies chronic inflammation and disease progression in heart failure: critical importance of the cardiosplenic axis. Circ Res. 2014;114:266-82.

19. Lynch TL 4th, Ismahil MA, Jegga AG, et al. Cardiac inflammation in genetic dilated cardiomyopathy caused by MYBPC3 mutation. J Mol Cell Cardiol. 2017;102:83-9.

20. Bozkurt B, Mann DL, Deswal A. Biomarkers of inflammation in heart failure. Heart Fail Rev. 2010;15:331-41.

21. Mann DL. Inflammatory mediators and the failing heart: past, present, and the foreseeable future. Circ Res. 2002;91:988-98.

22. Hamid T, Gu Y, Ortines RV, et al. Divergent tumor necrosis factor receptor-related remodeling responses in heart failure: role of nuclear factor-kappaB and inflammatory activation. Circulation. 2009;119:1386-97.

23. Braunwald E. Biomarkers in heart failure. N Engl J Med. 2008;358:2148-59.

24. Hulsmans M, Sam F, Nahrendorf M. Monocyte and macrophage contributions to cardiac remodeling. J Mol Cell Cardiol. 2015;93:149-55.

25. Karabacak M, Dogan A, Tayyar S, et al. Oxidative Stress Status Increase in Patients with Nonischemic Heart Failure. Med Princ Pract. 2014;23:532-7.

26. Belch JJF, Bridges $A B$, Scott $N$, et al. Oxygen free radicals and congestive heart failure. Br Heart J. 1991;65:245-8. 
27. McMurray J, Chopra M, Abdullah I, et al. Evidence of oxidative stress in chronic heart failure in humans. Eur Heart J. 1993;14:1493-8.

28. Walters AM, Porter GA Jr, Brookes PS. Mitochondria as a drug target in ischemic heart disease and cardiomyopathy. Circ Res. 2012;111:1222-36.

29. Stanley WC, Hoppel CL. Mitochondrial dysfunction in heart failure: potential for therapeutic interventions? Cardiovasc Res. 2000;45:805-6.

30. Yip H-K, Shao P-L, et al. Early intramyocardial implantation of exogenous mitochondria effectively preserved left ventricular function in doxorubicin-induced dilated cardiomyopathy rat. Am J Transl Res. 2020;12:4612-27.

31. Hu GW, Li Q, Niu X, et al. Exosomes secreted by human-induced pluripotent stem cell-derived mesenchymal stem cells attenuate limb ischemia by promoting angiogenesis in mice. Stem Cell Res Ther 2015 Apr 10;6:10.

32. Soontararak S, Chow L, Johnson V, et al. Mesenchymal Stem Cells (MSC) Derived from Induced Pluripotent Stem Cells (iPSC) Equivalent to Adipose-Derived MSC in Promoting Intestinal Healing and Microbiome Normalization in Mouse Inflammatory Bowel Disease Model. Stem Cells Transl Med. 2018;7:456-67.

33. Roux C, Saviane G, Pini J, et al. Immunosuppressive Mesenchymal Stromal Cells Derived from Human-Induced Pluripotent Stem Cells Induce Human Regulatory T Cells In Vitro and In Vivo. Front Immunol. 2018 Jan 25;8:1991.

34. Wu HJ, Yiu WH, Wong DWL, et al. Human induced pluripotent stem cell-derived mesenchymal stem cells prevent adriamycin nephropathy in mice. Oncotarget. 2017;8:103640-56.

35. Shafa M, lonescu LI, Vadivel A, et al. Human induced pluripotent stem cell-derived lung progenitor and alveolar epithelial cells attenuate hyperoxia-induced lung injury. Cytotherapy. 2018;20:108-25.

36. Ko SF, Yen-Ta Chen YT, Wallace CG, et al. Inducible Pluripotent Stem Cell-Derived Mesenchymal Stem Cell Therapy Effectively Protected Kidney from Acute Ischemia-Reperfusion Injury. Am J Transl Res. 2018;10:3053-67.

37. Lee MS, Lee FY, Chen YL, et al. Investigated the safety of intra-renal arterial transfusion of autologous CD34 + cells and time courses of creatinine levels, endothelial dysfunction biomarkers and micro-RNAs in chronic kidney disease patients-phase I clinical trial. Oncotarget. 2017;8:1775062.

38. Lee MS, Yip HK, Yang CC. el al. Overexpression of miR-19a and miR-20a in iPS-MSCs preserves renal function of chronic kidney disease with acute ischemia-reperfusion injury in rat. JCMM; 2021. (in press).

39. Lee FY, Chen KH, Wallace CG, et al. Xenogeneic human umbilical cord-derived mesenchymal stem cells reduce mortality in rats with acute respiratory distress syndrome complicated by sepsis. Oncotarget. 2017;8:45626-42.

40. Sun Pei-HsunSCheuk-Kwan, Sheung-Fat, et al. Impact of hyperglycemic control on left ventricular myocardium. A molecular and cellular basic study in a diabetic rat model. Int Heart J. 2009;50:191- 
206.

41. Lee F-Y, Chen Y-L, Sung P-H, et al. Intracoronary Transfusion of Circulation-Derived CD34 + Cells Improves Left Ventricular Function in Patients With End-Stage Diffuse Coronary Artery Disease Unsuitable for Coronary Intervention. Crit Care Med. 2015;43:2117-32.

42. Yip H-K, Fang W-F, Li Y-C, et al. Human Umbilical Cord-Derived Mesenchymal Stem Cells for Acute Respiratory Distress Syndrome. Crit Care Med. 2020;48:e391-9.

43. Chang Cheuk-KwanSLi-Teh, Sheu J-J, et al. Bone marrow-derived mononuclear cell therapy alleviates left ventricular remodeling and improves heart function in rat-dilated cardiomyopathy. Crit Care Med. 2009;37:1197-205.

44. Chua S, Lee F-Y, Chiang H-J, et al. The cardioprotective effect of melatonin and exendin-4 treatment in a rat model of cardiorenal syndrome. 2016; 61:438-456. J Pineal Res.

45. Yang $\mathrm{C}-\mathrm{C}$, Chen $\mathrm{Y}-\mathrm{T}$, et al. Early administration of empagliflozin preserved heart function in cardiorenal syndrome in rat. Biomed Pharmacother. 2019;109:658-70.

46. Yang $\mathrm{C}-\mathrm{H}$, Sheu J-J, Tsai T-H, et al. Effect of tacrolimus on myocardial infarction is associated with inflammation, ROS, MAP kinase and Akt pathways in mini-pigs. J Atheroscler Thromb. 2013;20(1):922.

\section{Figures}

(A)

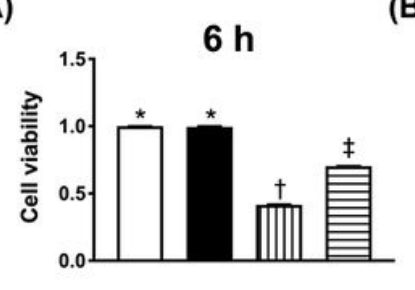

(D)

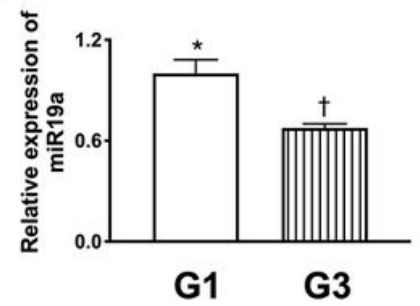

(B)

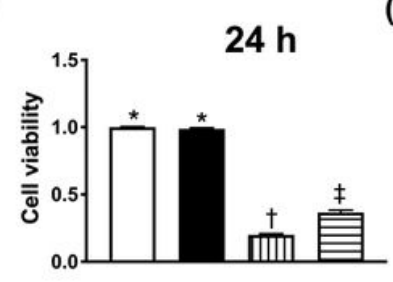

(E)

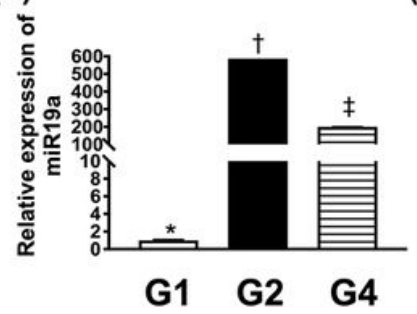

(C)

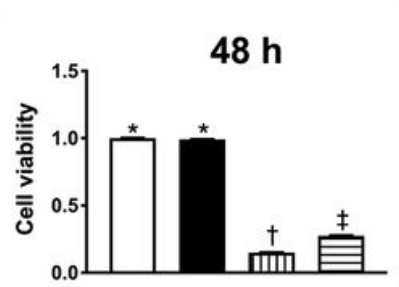

(F)

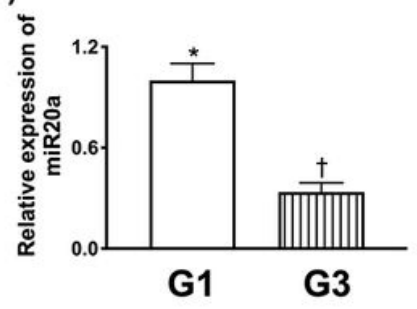

G1: iPS-MSC

G2: iPS-MSCdOex-mIRs

G3: iPS-MSC $+\mathrm{H}_{2} \mathrm{O}_{2} / 100 \mu \mathrm{M}$

G4: iPS-MSCdOex-mIRs+ $\mathrm{H}_{2} \mathrm{O}_{2} / 100 \mu \mathrm{M}$

(G)

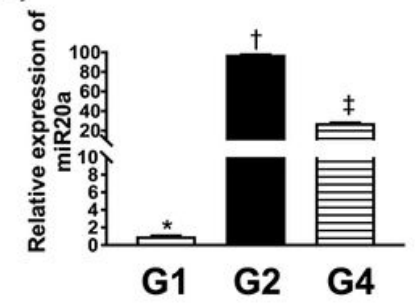

Figure 1

MTT assay for evaluating the impact of double overexpression of microRNAs on against the oxidative stress damage and GPCR A) By $6 \mathrm{~h}$ after cell culturing, the result of MTT assay, * vs. other groups with different symbols $(t, \ddagger), p<0.0001$. B) By $24 \mathrm{~h}$ after cell culturing, the result of MTT assay, * vs. other groups with different symbols $(\dagger, \ddagger), p<0.0001$. C) By $48 \mathrm{~h}$ after cell culturing, the result of MTT assay, * vs. other groups with different symbols $(\dagger, \ddagger), p<0.0001$. $n=8$ for each group. $G 1=i P S-M S C ; G 2=i P S-$ 
MSCdOex-mIRs; G3 = iPS-MSC + H202/100uM; G4 = iPS-MSCdOex-mIRs + H2O2/100uM. D) The qPCR analytical result of relative expression of miR-19a-3p in iPS-MSC with and without $\mathrm{H} 2 \mathrm{O} 2$ treatment, * vs. $t, p<0.01$. E) The $q P C R$ analytical result of relative expression of miR-19a-3p in iPS-MSCdOex-mIRs with and without $\mathrm{H} 2 \mathrm{O} 2$ treatment, * vs. other groups with different symbols $(t, \ddagger), p<0.0001$. F) The qPCR analytical result of relative expression of miR-20a-5p in iPS-MSC with and without $\mathrm{H} 2 \mathrm{O} 2$ treatment, * vs. $t, p<0.01$. G) The qPCR analytical result of relative expression of miR-20a-5p in iPS-MSCdOex-mIRs with and without $\mathrm{H} 2 \mathrm{O} 2$ treatment, * vs. other groups with different symbols $(t, \ddagger), p<0.0001$. $n=4$ for each group. dOex-mIRs = overexpression of double microRNAs (i.e., miR-19a-3p and miR-20a-5p). All statistical analyses were performed by one-way ANOVA, followed by Bonferroni multiple comparison post hoc test. Symbols $(*,+, \ddagger)$ indicate significance (at 0.05 level).

(A)

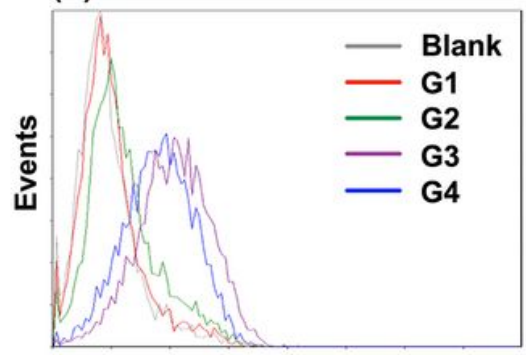

H2DCFDA

(C)

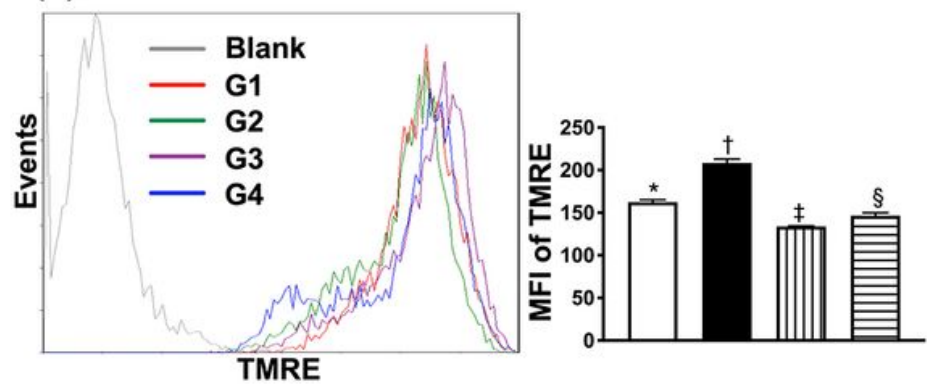

(B)

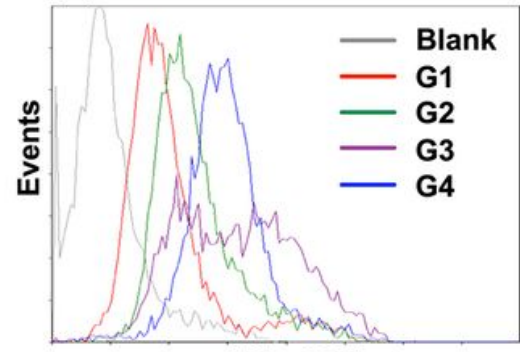

Mito-SOX
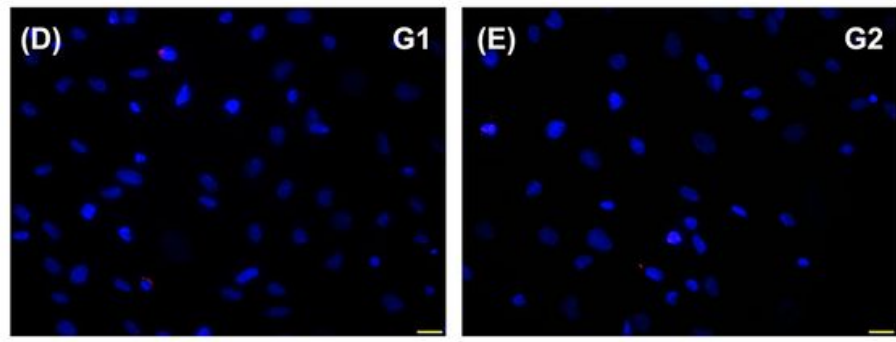
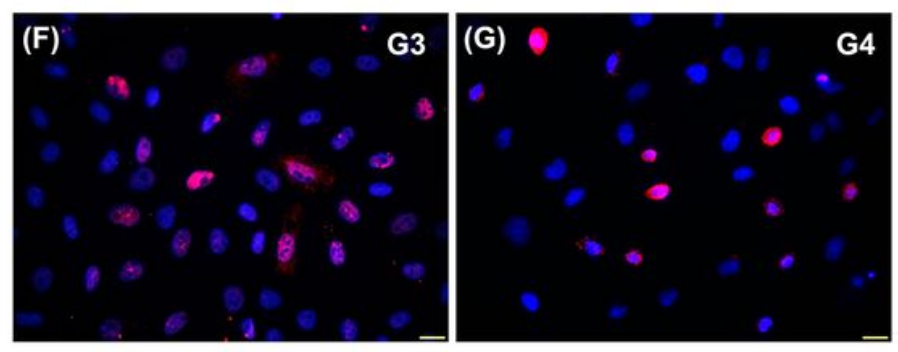

(H)

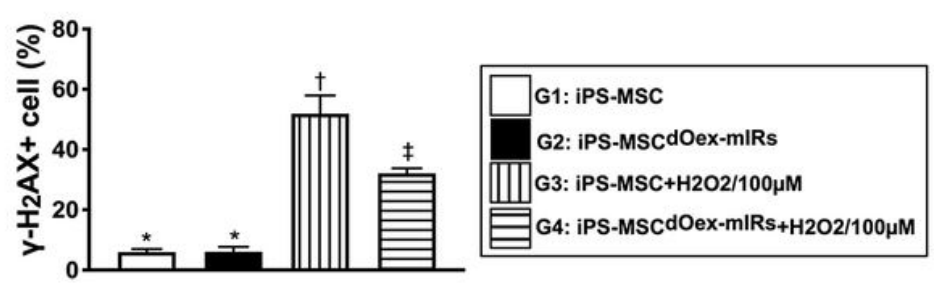

\section{Figure 2}

Flow cytometric analysis of oxidative stress and mitochondrial membrane potential in iPS-MSCs and iPSMSCdOex-mIRs and IF microscopic findings A) Fluorescent intensity of DCFDA (i.e., an indicator of total intracellular oxidative stress), * vs. other groups with different symbols $(t, \ddagger), p<0.0001$. B) Fluorescent intensity of Mito-SOX (i.e., indicator of mitochondrial oxidative stress), * vs. other groups with different symbols $(t, \ddagger), p<0.0001$. C) Fluorescent intensity of TMRE (i.e., an index mitochondrial membrane potential), * vs. other groups with different symbols $(\dagger, \ddagger, \S), p<0.0001$. $n=8$ for each group. $D$ to $G)$ Illustrating the immunofluorescent microscopic finding (400x) for identification of $\mathrm{y}-\mathrm{H} 2 \mathrm{AX}+$ cells (pink 
color). H) Analytical result of positively stained $\mathrm{y}-\mathrm{H} 2 \mathrm{AX}$ cells, * $v s$. other groups with different symbols ( $\mathrm{t}$, $\ddagger), p<0.001$. $n=4$ for each group. Scale bars in lower right corner represent $20 \mu \mathrm{m}$. All statistical analyses were performed by one-way ANOVA, followed by Bonferroni multiple comparison post hoc test. Symbols $(*, \dagger, \ddagger)$ indicate significance (at 0.05 level). MFI = mean fluorescent intensity; G1 = iPS-MSC; G2 = iPSMSCdOex-mIRs; G3 = iPS-MSC + H2O2/100uM; G4 = iPS-MSCdOex-mIRs + H2O2/100uM.

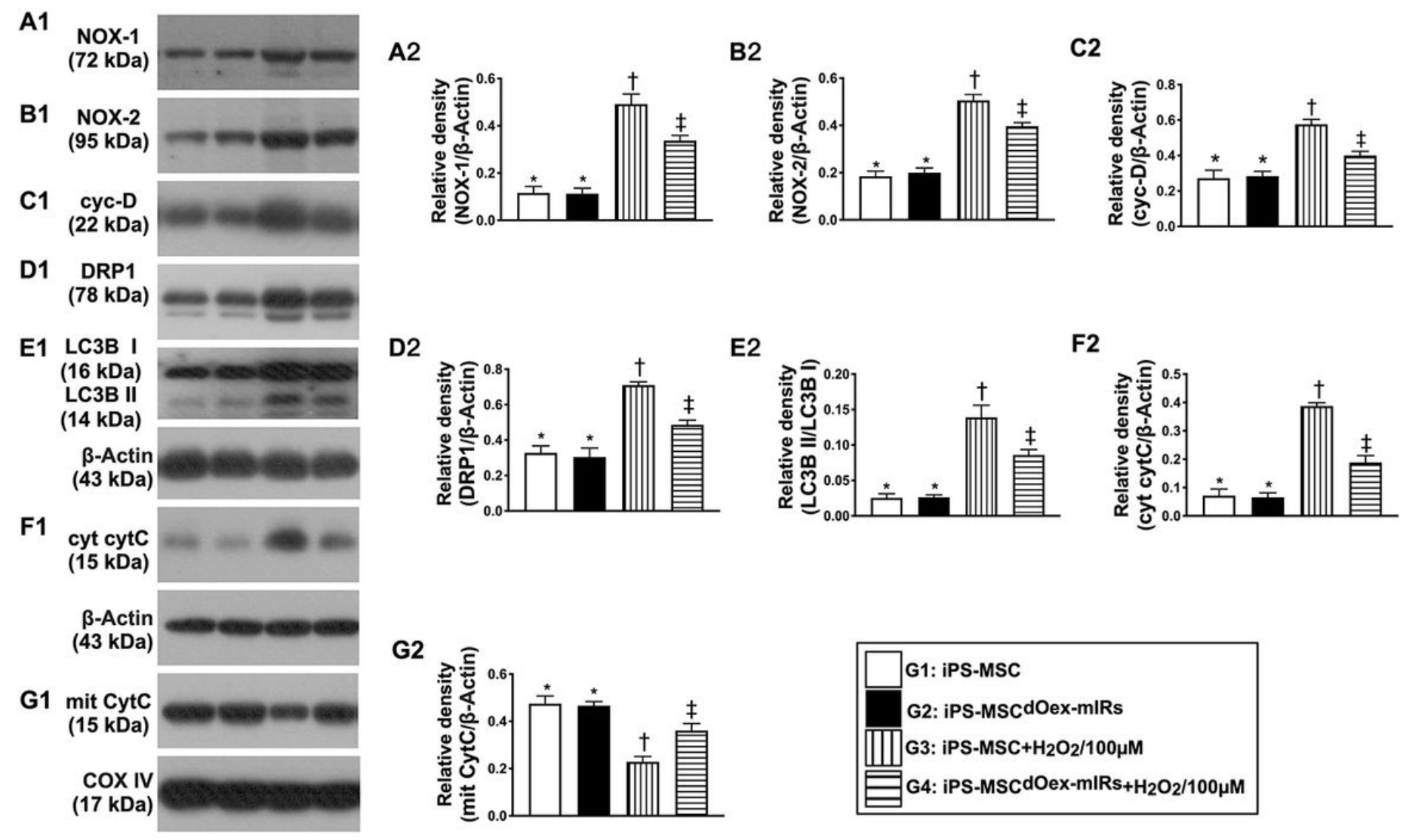

Figure 3

In vitro analysis of protein expressions of oxidative stress A1, A2) Protein expression of NOX-1, * vs. other groups with different symbols $(t, \ddagger), p<0.001$. B1, B2) Protein expression of NOX-2, * vs. other groups with different symbols $(+, \ddagger), p<0.001$. C1, C2) Protein expression of cyclophilin $D(c y c-D)$, * vs. other groups with different symbols $(\dagger, \ddagger), p<0.001$. D1, D2) Protein expression of and dynamin related protein 1 (DRP1), * vs. other groups with different symbols ( $t, \ddagger), p<0.001$. E1, E2) Protein expression of ratio of LC3B-II to LC3B-I, * vs. other groups with different symbols $(t, \ddagger), p<0.001$. F1, F2) Protein expression of cytosolic cytochrome $C$ (cyt-cytC), * vs. other groups with different symbols ( $\dagger, \ddagger), p<0.001$ (Actin). G1, G2) Protein expression of mitochondrial cytochrome C (mit-cytC), * vs. other groups with different symbols $(\dagger, \ddagger), p<0.001$. All statistical analyses were performed by one-way ANOVA, followed by Bonferroni multiple comparison post hoc test $(n=3)$. Symbols $\left({ }^{*}, t, \neq\right)$ indicate significance (at 0.05 level). G1 = iPS-MSC; G2 = iPS-MSCdOex-mIRs; G3 = iPS-MSC + H2O2/100uM; G4 = iPS-MSCdOex-mIRs + $\mathrm{H} 2 \mathrm{O} 2 / 100 \mathrm{uM}$. 

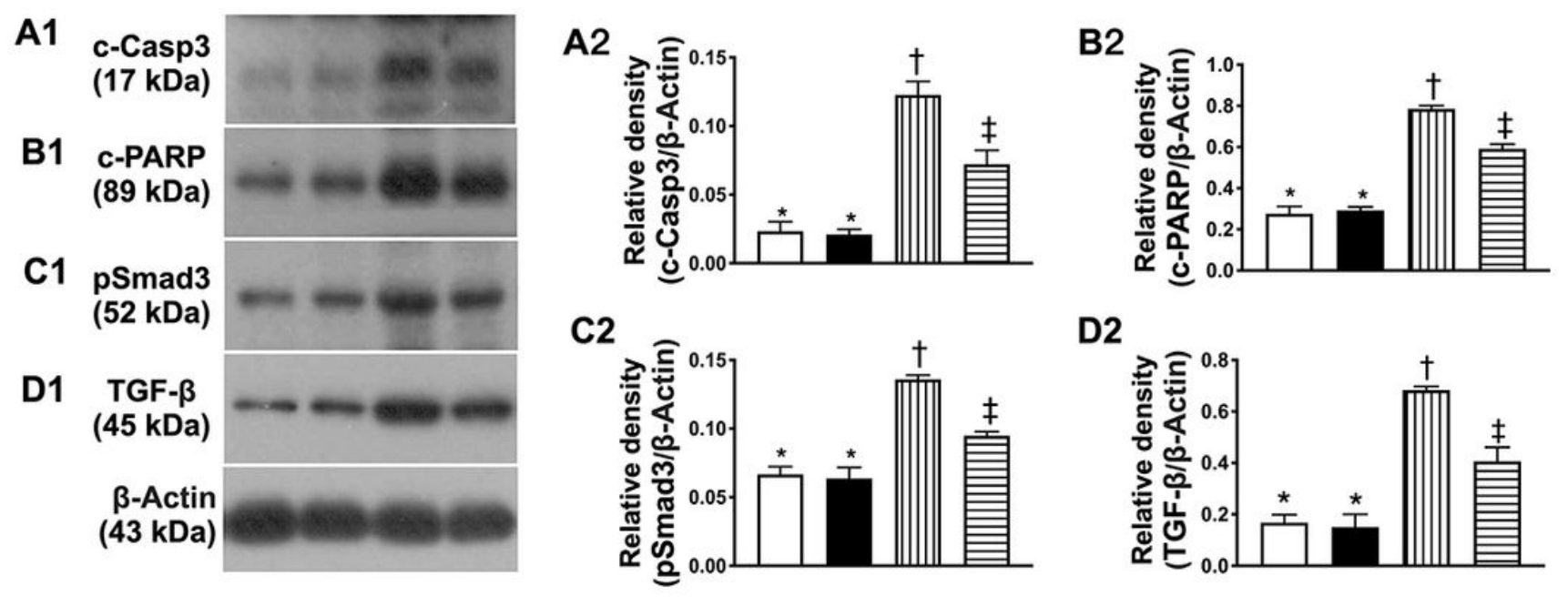

D2
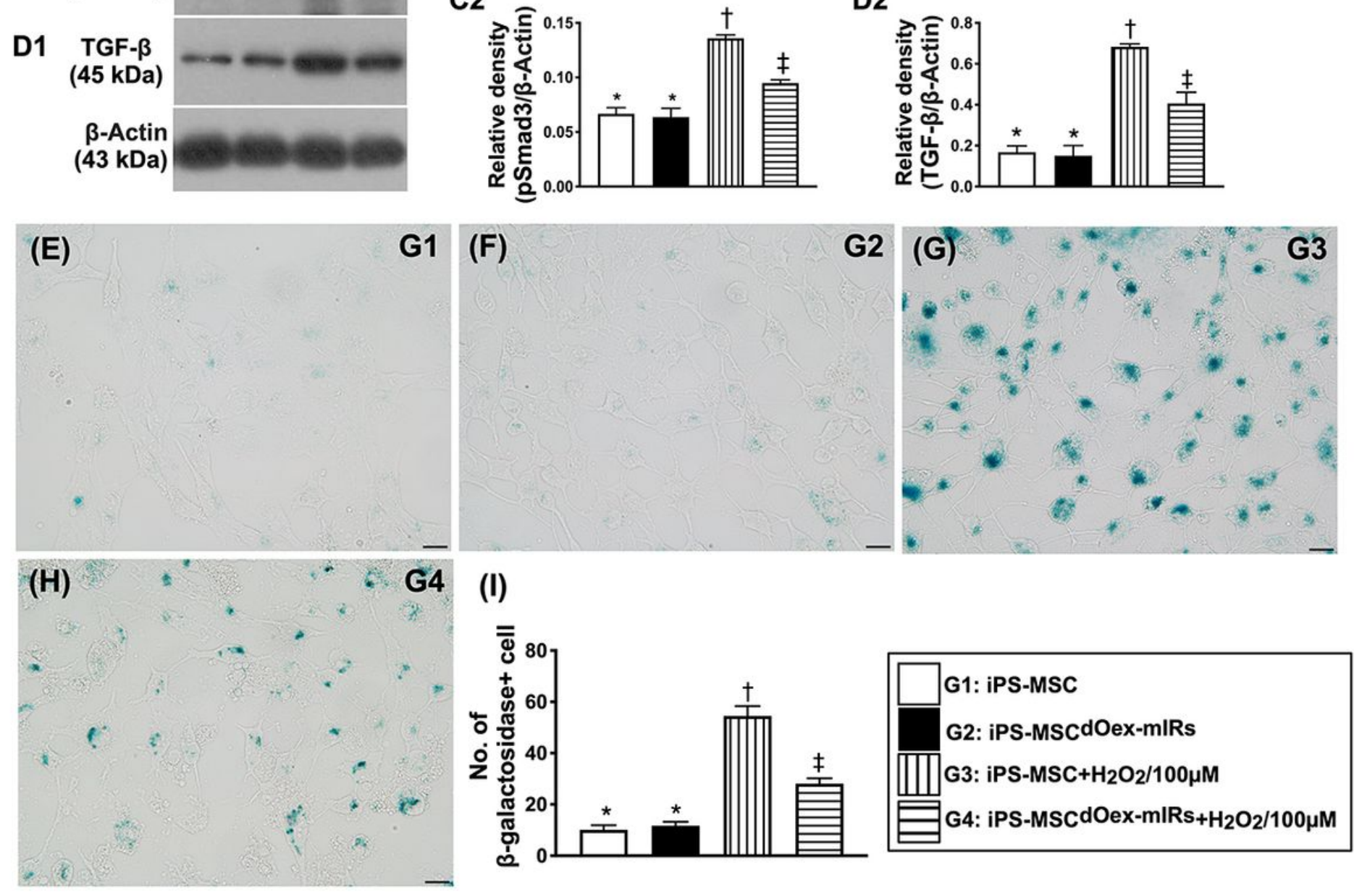

\section{Figure 4}

In vitro analysis of protein expressions of apoptosis and fibrosis cellular level of senescence A1, A2) Protein expression of cleaved caspase 3 (c-Casp3), * vs. other groups with different symbols $(\dagger, \ddagger)$, p<0.001. B1, B2) Protein expression of cleaved Poly (ADP-ribose) polymerase (c-PARP), * vs. other groups with different symbols $(\dagger, \ddagger), p<0.001$. C1, C2) Protein expression of Smad3, * vs. other groups with different symbols $(\dagger, \ddagger), p<0.001$. D1, D2) Transforming growth factor (TGF) $-\beta$, * vs. other groups with different symbols $(\dagger, \ddagger), p<0.001$. $E$ to $H)$ Illustrating the immunofluorescent microscopic finding (400x) for identification of positively-stained $\beta$-galactosidase cells (blue color). I) Analytical result of number of $\beta$-galactosidase + cells, * vs. other groups with different symbols $(t, \ddagger), p<0.001$. Scale bars in lower right corner represent $20 \mu \mathrm{m}$. All statistical analyses were performed by one-way ANOVA, followed by Bonferroni multiple comparison post hoc test $(n=4)$. Symbols $(*,+, \neq)$ indicate significance (at 0.05 level). 
G1 = iPS-MSC); G2 = iPS-MSCdOex-mIRs; G3 = iPS-MSC + H202/100uM; G4 = iPS-MSCdOex-mIRs + H2O2/100uM.

(A)
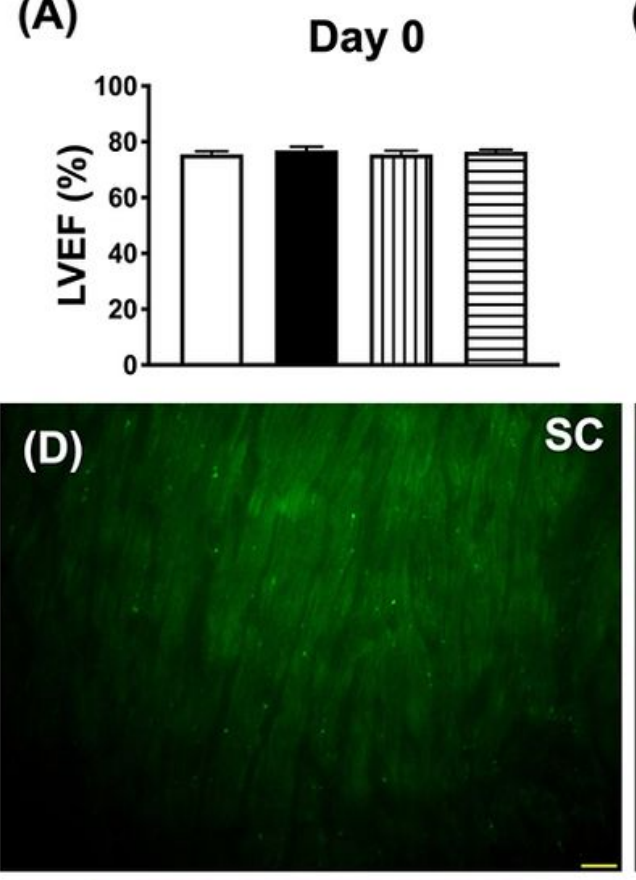

(G) DCM+iPS-MSCdOex-mIRs
(B)
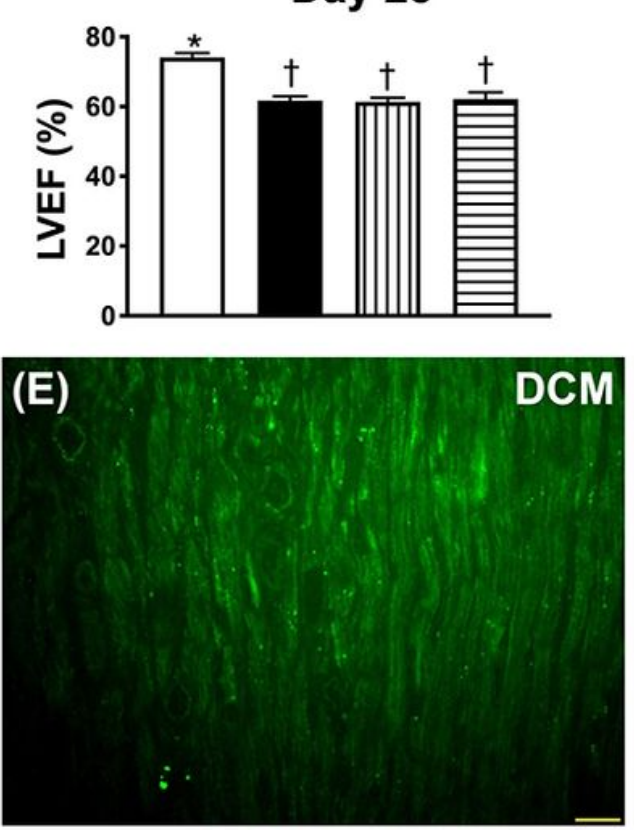

(H)
(C)

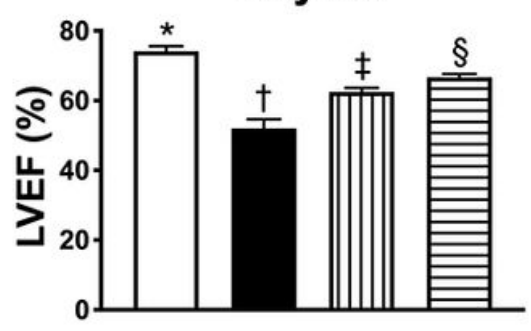

(F)

DCM+iPS-MSC
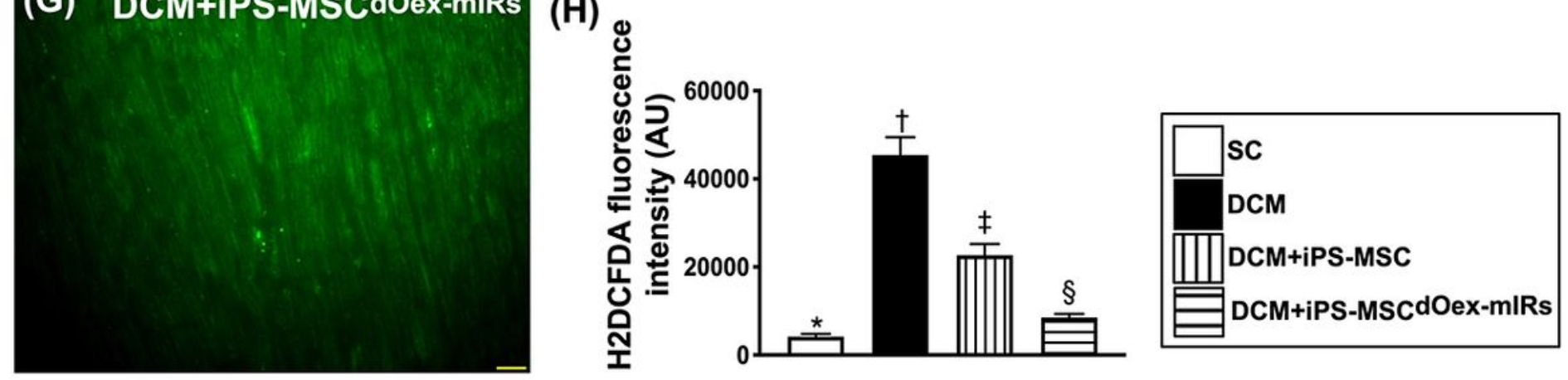

Figure 5

Serial changes of LVEF and fluorescent intensity of oxidative stress in LV myocardium by day 60 after DCM induction A) By day 0 prior to DCM induction, analytical result of the LVEF, p>0.5. B) By day 28 after DCM induction, * vs. $\dagger, p<0.0001$. C) By day 60 after DCM induction, * vs. other groups with different symbols $(t, \ddagger, \S), p<0.0001$. $n=8$ for each group. $D$ to $G)$ Illustrating the immunofluorescent microscopic finding (400x) for evaluation of fluorescent intensity (i.e., H2DCFDA assay) of oxidative stress in living myocardium (green color). H) Analytical result of fluorescent intensity of H2DCFDA staining in LV myocardium, * vs. other groups with different symbols $(t, \neq, \S), p<0.0001$. Scale bars in lower right corner represent $20 \mu \mathrm{m} . \mathrm{n}=4$ for each group. All statistical analyses were performed by one-way ANOVA, followed by Bonferroni multiple comparison post hoc test. Symbols $(*,+, \neq, \S)$ indicate significance (at 0.05 level). LVEF = left ventricular ejection fraction; DCM = dilated cardiomyopathy; iPS-MSCs = inducible pluripotent stem cell-derived mesenchymal stem cells; dOex-mIRs = double overexpression of microRNAs. 

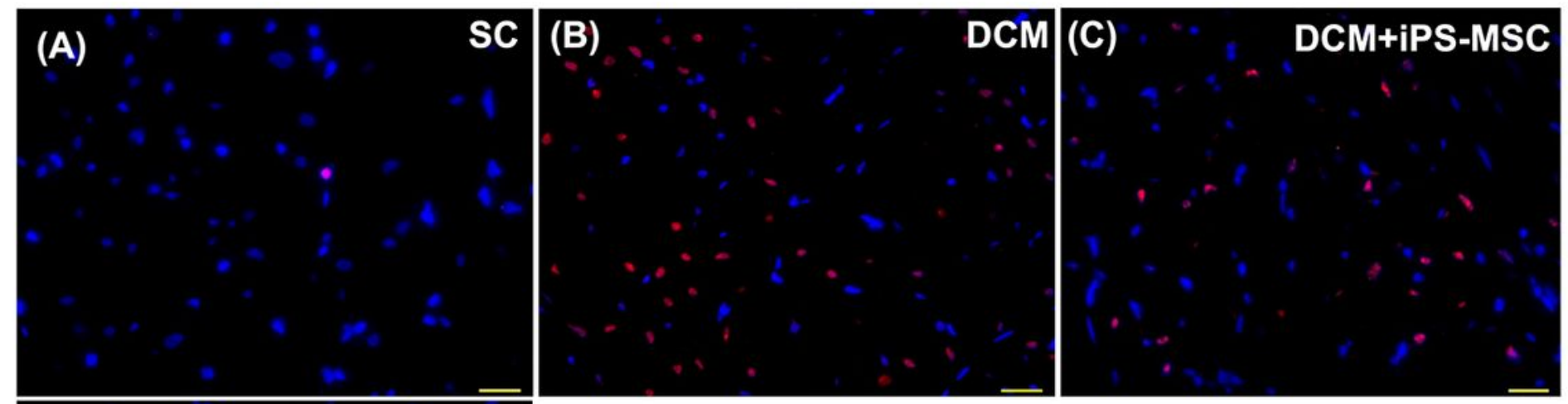

(D) DCM+iPS-MSCdOex-mIRs

\section{(E)}
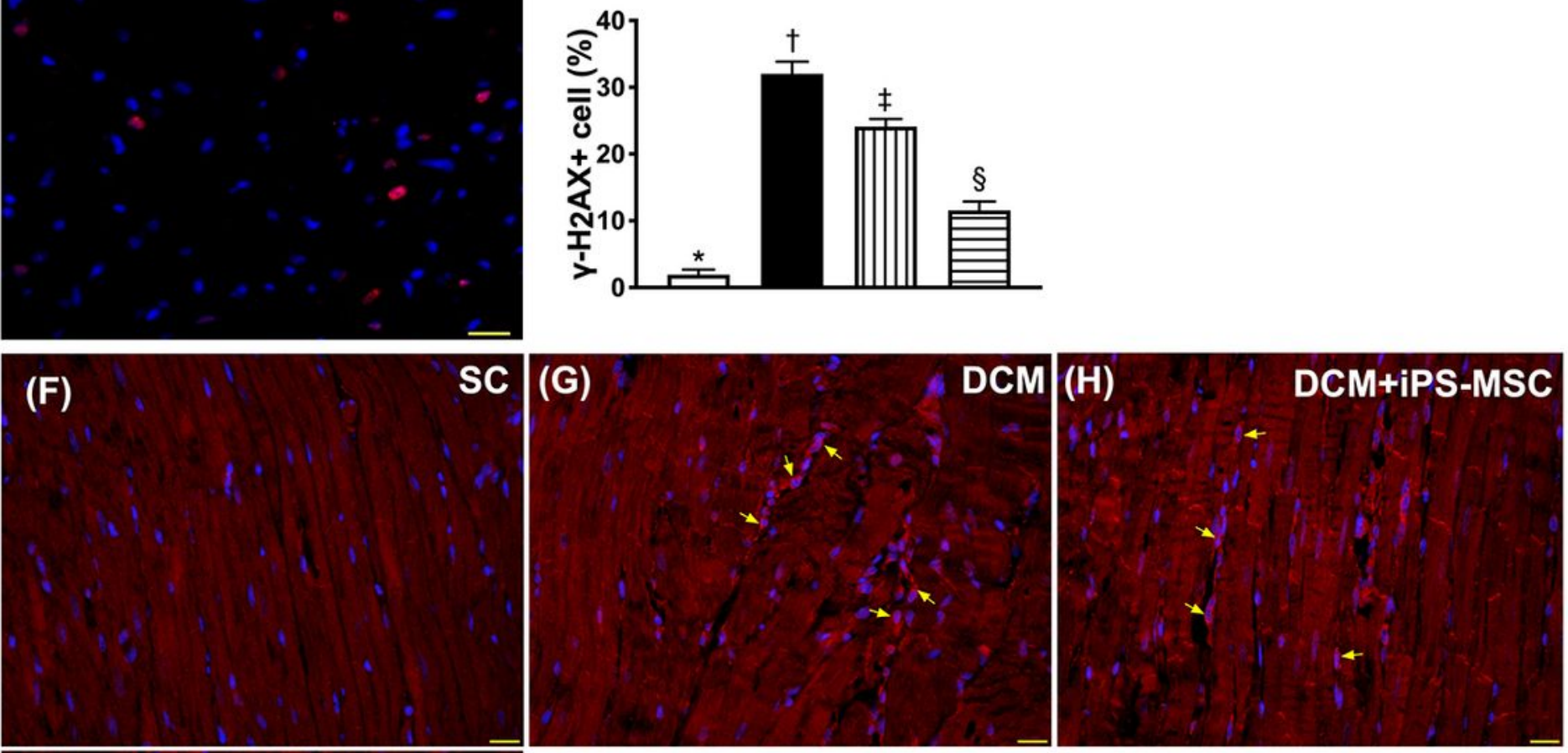

(I) DCM+iPS-MSCdOex-mIRs

\section{(J)}
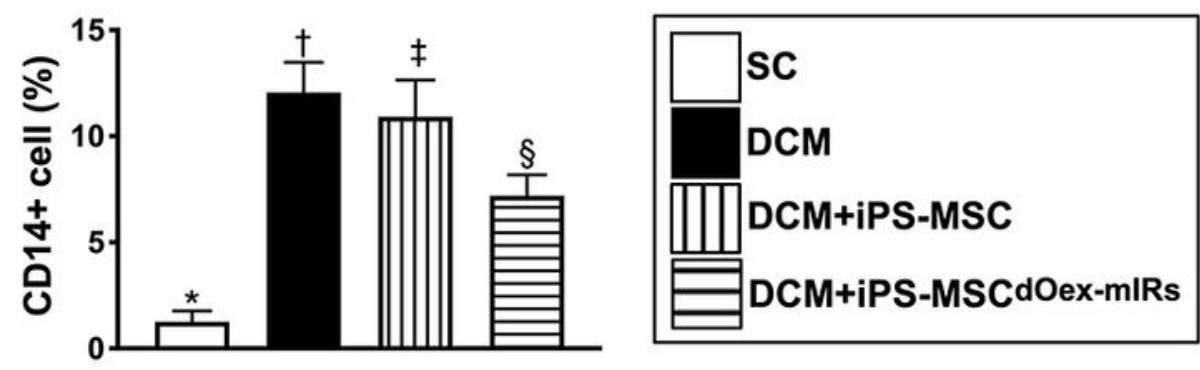

\section{Figure 6}

Cellular levels of DNA-damaged and inflammatory biomarkers in LV myocardium by day 60 after DCM induction $A$ to $D$ ) Illustrating the immunofluorescent (IF) microscopic finding for identification the expressions of $\mathrm{y}-\mathrm{H} 2 \mathrm{AX}+$ cells (pink color). E) Analytical result of number of $\mathrm{Y}-\mathrm{H} 2 \mathrm{AX}+$ cells, * vs. other groups with different symbols $(t, \neq, \S), p<0.0001$. F to I) Showing the IF microscopic finding for identification of CD14+ cells (green color). J) Analytical result of number of CD14+ cells, * vs. other 
groups with different symbols $(\dagger, \ddagger, \S), p<0.0001$. Scale bars in lower right corner represent $20 \mu \mathrm{m}$. All statistical analyses were performed by one-way ANOVA, followed by Bonferroni multiple comparison post hoc test ( $n=6$ for each group). Symbols $(*,+, \neq, \S)$ indicate significance (at 0.05 level). LVEF $=$ left ventricular ejection fraction; $\mathrm{DCM}=$ dilated cardiomyopathy; iPS-MSCs $=$ inducible pluripotent stem cellderived mesenchymal stem cells; dOex-mIRs = double overexpression of microRNAs.

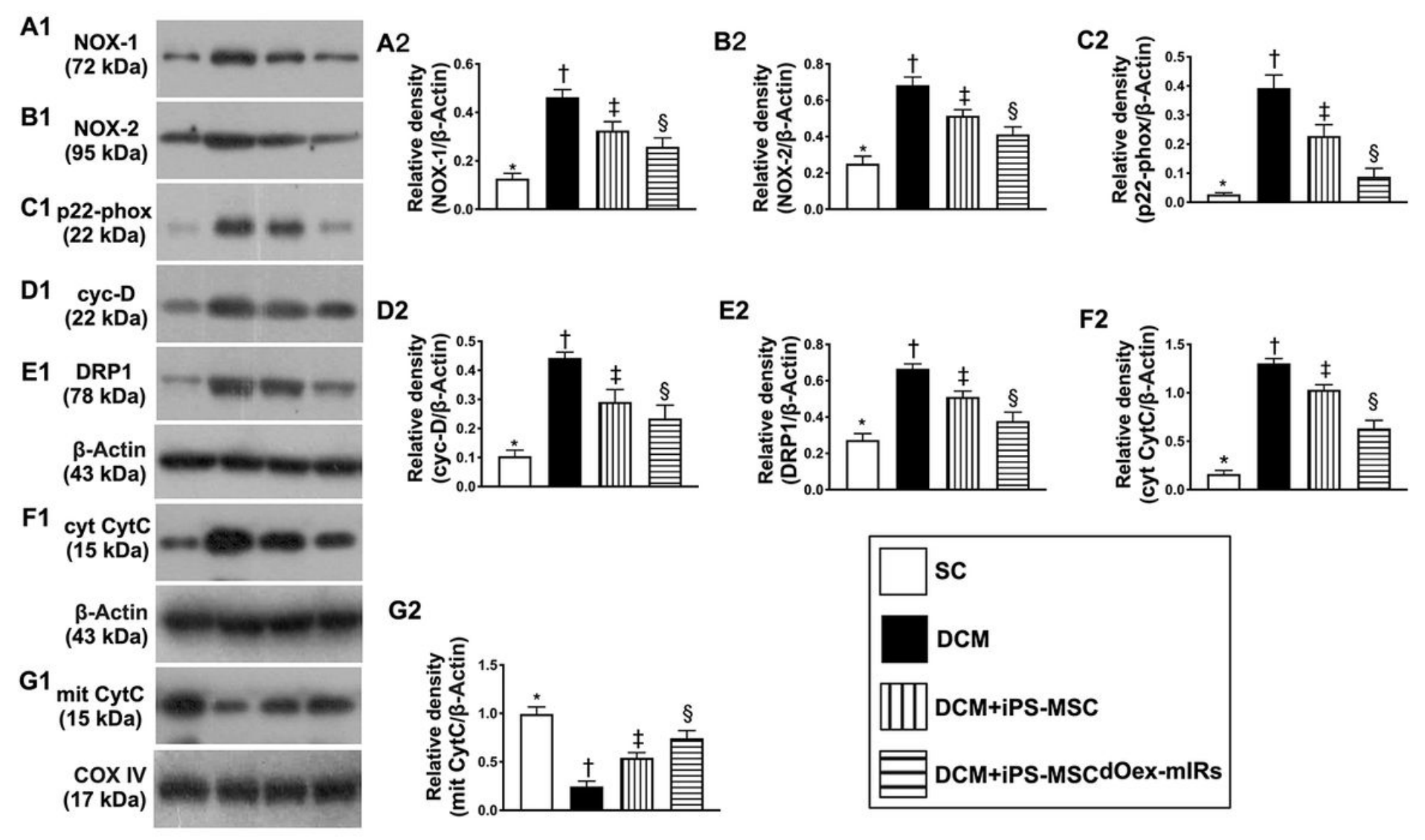

Figure 7

Protein expressions of oxidative stress and mitochondrial damaged biomarkers in LV myocardium by day 60 after DCM induction A1, A2) Protein expression of NOX-1, * vs. other groups with different symbols ( $\dagger$, $\ddagger, \S), \mathrm{p}<0.0001$. B1, B2) Protein expression of NXO-2, * vs. other groups with different symbols $(t, \ddagger, \S)$, $p<0.0001 . C 1, C 2)$ Protein expression of p22 phox, * vs. other groups with different symbols $(\dagger, \ddagger, \S)$, $p<0.0001$. D1, D2) Protein expression of cyclophilin D (cyc-D), * vs. other groups with different symbols $(t, \neq, \S), p<0.0001$. E1, E2) Protein expression of DRP1, * vs. other groups with different symbols $(t, \neq, \S)$, $p<0.0001$. F1, F2) Protein expression of cytosolic cytochrome $C$ (cyt-CytC), * vs. other groups with different symbols $(\dagger, \ddagger, \S), p<0.0001$. G1, G2) Protein expression of mitochondrial cytochrome $C$ (mitCytC), * vs. other groups with different symbols $(t, \neq, \S), p<0.0001$. All statistical analyses were performed by one-way ANOVA, followed by Bonferroni multiple comparison post hoc test ( $\mathrm{n}=6$ for each group). Symbols $(*, \dagger, \ddagger$ §) indicate significance (at 0.05 level). LVEF = left ventricular ejection fraction; DCM = dilated cardiomyopathy; iPS-MSCs = inducible pluripotent stem cell-derived mesenchymal stem cells; dOex-mIRs $=$ double overexpression of microRNAs. 


\section{DCM}

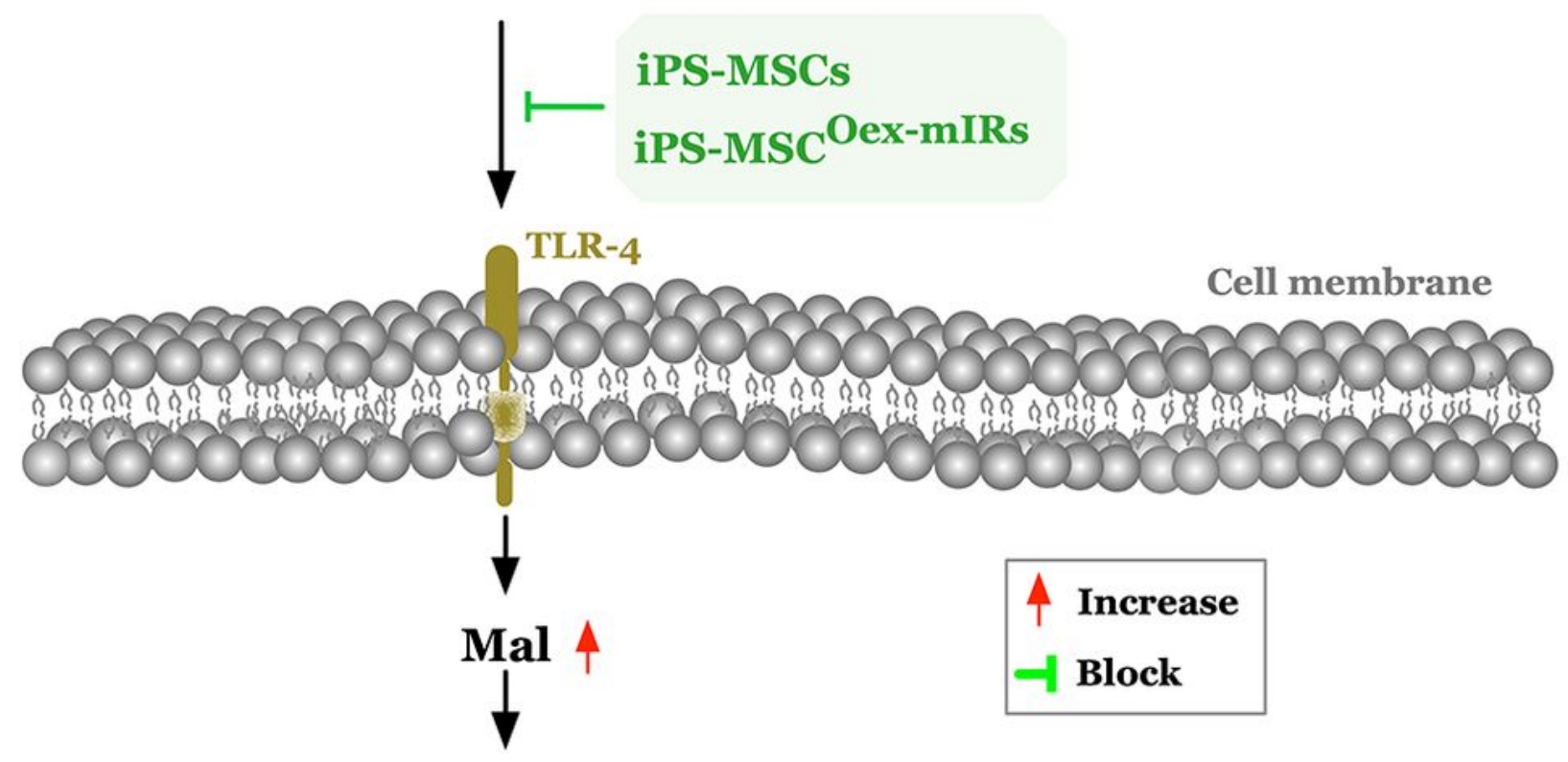

MyD88 个<smiles>[CH]1CCC1</smiles>

TRAF6 $\uparrow$<smiles>C[13CH]</smiles>

IKK complex $(\boldsymbol{\alpha}, \boldsymbol{\beta}) \uparrow$<smiles>C1CC1</smiles>

IKB-a 4<smiles>[3H][AlH2]</smiles>

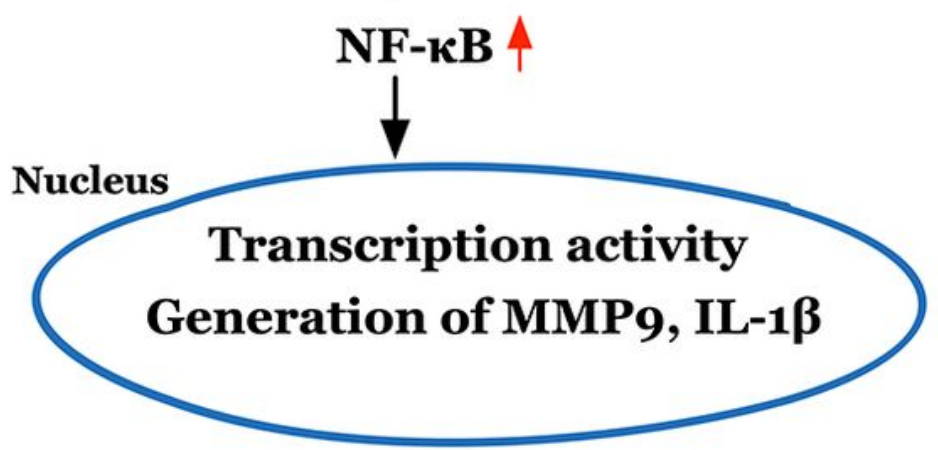

Figure 8

Schematically illustrated the underlying mechanisms of oxidative-stress and its downstream signaling that involved in the myocardial damage in DCM setting DCM = dilated cardiomyopathy; ROS = reactive oxygen species; iPS-MSCs = inducible pluripotent stem cell-derived mesenchymal stem cells; dOex-mIRs = double overexpression of microRNAs. 

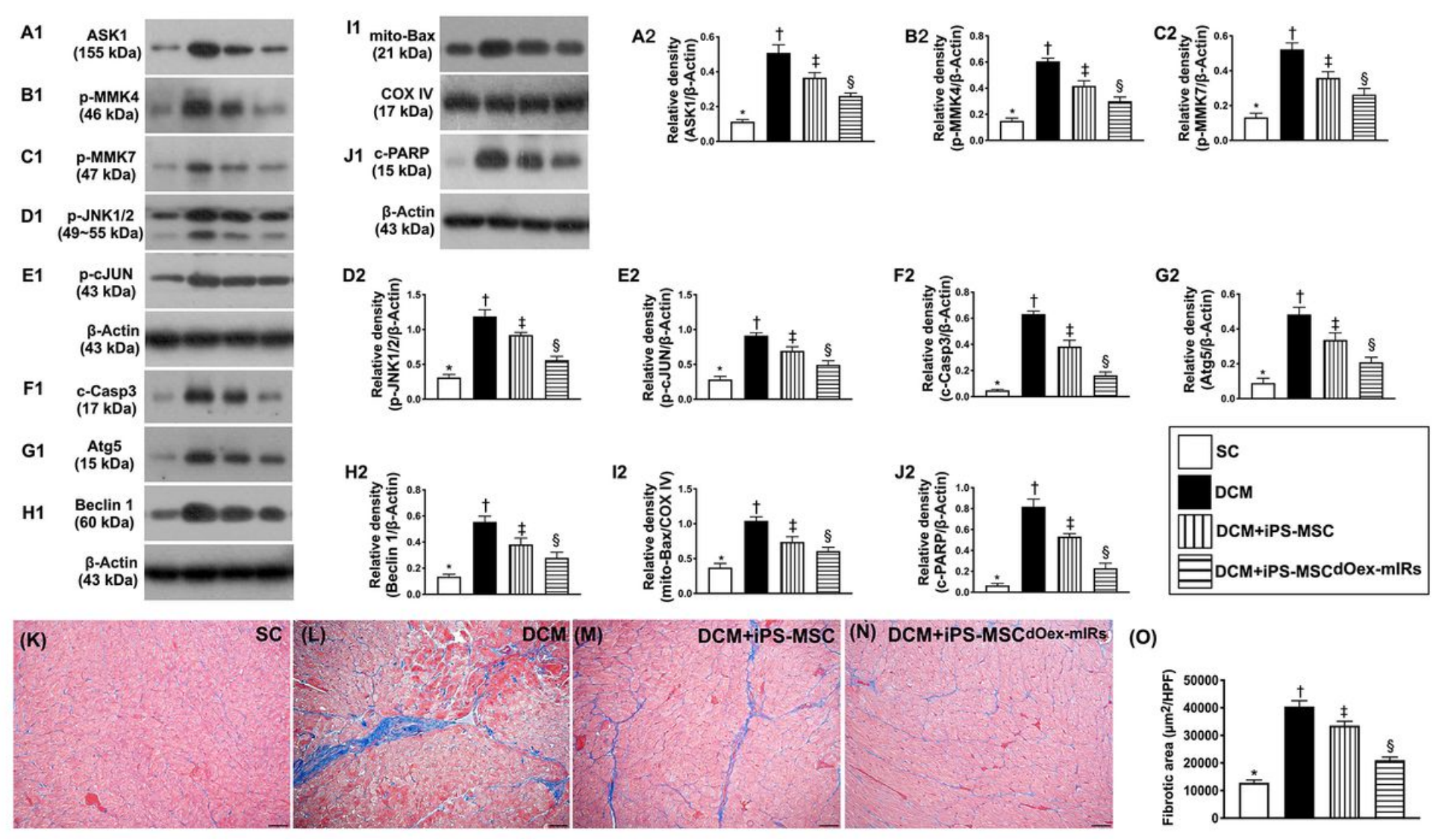

\section{Figure 9}

Protein expressions of mitogen-activated protein kinase (MAPK) pathway, apoptosis, autophagic and fibrotic biomarkers in LV myocardium by day 60 after DCM induction A1, A2) Protein expression of apoptosis signal-regulating kinase 1 (ASK1), * vs. other groups with different symbols $(\dagger, \ddagger, \S), p<0.0001$. B1, B2) Protein expression of phosphorylated mitogen-activated protein kinase 4 (p-MMK4), * vs. other groups with different symbols $(\dagger, \ddagger, \S), \mathrm{p}<0.0001$. C1, C2) Protein expression of $\mathrm{p}-\mathrm{MMK} 7$, * vs. other groups with different symbols $(\dagger, \ddagger, \S), p<0.0001$. D1, D2) Protein expression of $p-J N K 1 / 2$, * vs. other groups with different symbols $(t, \ddagger, \S), p<0.0001$. E1, E2) Protein expression of $p$-cJUN, * vs. other groups with different symbols ( $t, \ddagger, \S), p<0.0001$. F1, F2) Protein expression of cleaved caspase 3 (c-Casp3), * vs. other groups with different symbols $(t, \neq, \S), p<0.0001$. G1, G2) Protein expression of Atg5, * vs. other groups with different symbols $(t, \neq, \S), p<0.0001 . H 1, H 2)$ Protein expression of Beclin1, * vs. other groups with different symbols $(t, \ddagger, \S), p<0.0001$. 11, 12) Protein expression of mitochondrial Bax (mito-Bax), vs. other groups with different symbols $(t, \neq, \S), p<0.0001$. J1, J2) Protein expression of cleaved Poly (ADPribose) polymerase (c-PARP), * vs. other groups with different symbols $(\dagger, \ddagger, \S), p<0.0001$. K to $N)$ Illustrating microscopic finding (200x) of the Masson's trichrome stain for identification of fibrotic area in LV myocardium (blue color). 0) Analytical result of fibrotic area, * vs. other groups with different symbols $(t, \neq, \S), p<0.0001$. Scale bars in lower right corner represent $50 \mu \mathrm{m}$. All statistical analyses were performed by one-way ANOVA, followed by Bonferroni multiple comparison post hoc test $(n=6$ for each group). Symbols $(*, \dagger, \ddagger \S \S)$ indicate significance (at 0.05 level). LVEF = left ventricular ejection fraction; 
DCM = dilated cardiomyopathy; iPS-MSCs = inducible pluripotent stem cell-derived mesenchymal stem cells; dOex-mIRs = double overexpression of microRNAs.
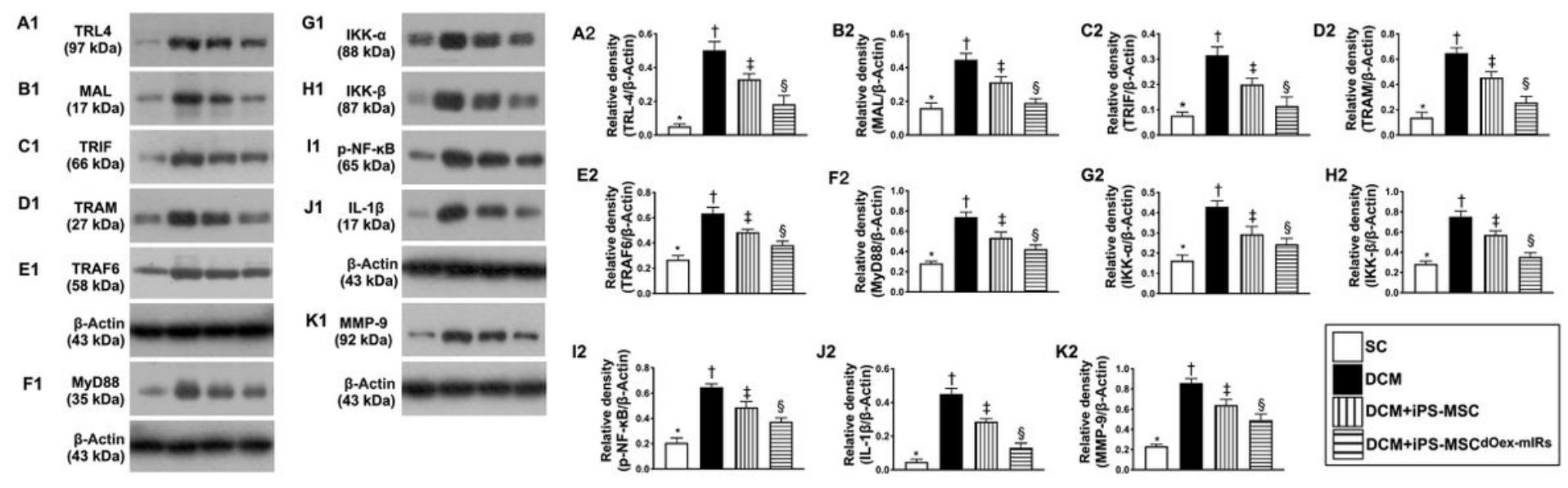

\section{Figure 10}

Protein expressions of upstream and downstream inflammatory signaling pathways in LV myocardium by day 60 after DCM induction A1, A2) Protein expression of toll-like receptor 4 (TLR4), * vs. other groups with different symbols $(t, \neq, \S), p<0.0001$. B1, B2) MyD88 adaptor-like (MAL), * vs. other groups with different symbols $(\dagger, \ddagger, \S), p<0.0001$. C1, C2) Protein expression of Toll/IL-1R domain-containing adaptorinducing IFN- $\beta$ (TRIF), * vs. other groups with different symbols $(t, \neq, \S), p<0.0001$. D1, D2) Translocating chain-associated membrane protein (TRAM), * vs. other groups with different symbols $(\dagger, \ddagger, \S), p<0.0001$. E1, E2) Protein expression of TNF receptor associated factor 6 (TRAF6), * vs. other groups with different symbols $(\dagger, \ddagger, \S), p<0.0001$. F1, F2) Protein expression of myeloid differentiation primary response 88 (MyD88), * vs. other groups with different symbols ( $\dagger, \ddagger, \S), p<0.0001 . \mathrm{G} 1, \mathrm{G} 2)$ Protein expression of IKB Kinase a (IKK-a), * vs. other groups with different symbols $(t, \neq, \S), p<0.0001$. H1, H2) Protein expression of IKK- $\beta$, * vs. other groups with different symbols $(\dagger, \ddagger, \S), p<0.0001$. I1, 12) Protein expression of phosphorylated nuclear factor $ß$ ( $p-N F-K ß)$, * vs. other groups with different symbols $(t, \neq, \S), p<0.0001$. $J 1, J 2)$ Protein expressions of interleukin (IL)-1ß, * vs. other groups with different symbols $(\dagger, \ddagger$, §), $p<0.0001$. K1, K2) Protein expression of matrix metalloproteinase (MMP)-9, * vs. other groups with different symbols $(t, \neq, \S), p<0.0001$. All statistical analyses were performed by one-way ANOVA, followed by Bonferroni multiple comparison post hoc test ( $n=6$ for each group). Symbols $(*,+, \neq, \S)$ indicate significance (at 0.05 level). LVEF = left ventricular ejection fraction; DCM = dilated cardiomyopathy; iPSMSCs = inducible pluripotent stem cell-derived mesenchymal stem cells; dOex-mIRs = double overexpression of microRNAs. 


\section{DCM$$
\downarrow \vdash \begin{aligned}
& \text { iPS-MSCs } \\
& \text { iPS-MSC }
\end{aligned}
$$ \\ ROS generation $\uparrow$ \\ $\downarrow$ \\ NADPH oxidase $\downarrow$}

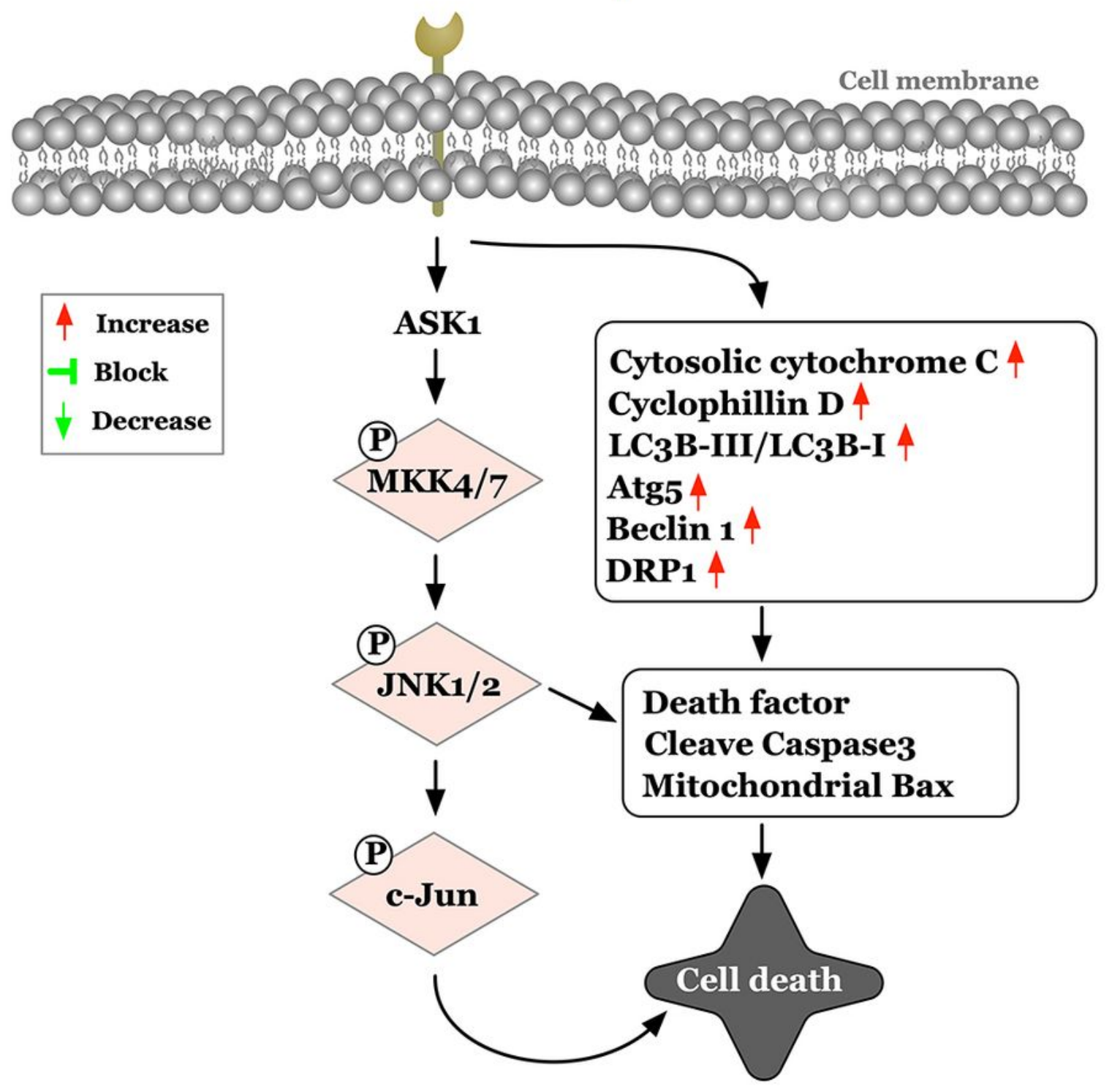

Figure 11

Schematically illustrate the upstream and downstream inflammatory signaling pathways on DCM rodent DCM = dilated cardiomyopathy; iPS-MSCs = inducible pluripotent stem cell-derived mesenchymal stem cells; dOex-mIRs = double overexpression of microRNAs.

\section{Supplementary Files}


This is a list of supplementary files associated with this preprint. Click to download.

- SupplementrayFigure1.jpg 